\title{
18. EOCENE THROUGH QUATERNARY PLANKTONIC FORAMINIFERS FROM THE NORTHWEST PACIFIC, LEG 126 $^{1}$
}

\author{
Yvonne Herman ${ }^{2}$
}

\begin{abstract}
Eocene through Quaternary planktonic foraminifers were identified in cores recovered during Leg 126. Turbidites and volcanic ash beds are intercalated with hemipelagic sediments. Preservation of foraminifers is variable, ranging from excellent to poor and appears to have been affected by fluctuations in the carbonate compensation depth (CCD), depth of burial, changes in bottom water temperature, current velocity, sediment accumulation rates and seafloor topography. Preservation of foraminifers in Quaternary sediments is generally good, however, species abundance varies by a factor of $10^{5}-10^{6}$ and reflects dilution by volcanogenic as well as terrigenous constituents and cannot be used for paleoceanographic reconstructions. In pre-Quaternary deposits planktonic foraminiferal tests frequently exhibit dissolution effects; biostratigraphic zonation and placement of zonal boundaries is difficult owing to hiatuses, dissolution facies, extraneously deposited sediments, and discontinuous coring. The Eocene foraminiferal faunas include specimens of the Globorotalia cerroazulensis plexus, markers of Zone P16 as well as Globigerina senni and Globigerinatheka spp., which became extinct before the end of the Eocene. Six hiatuses and/or dissolution periods, probably reflecting global cooling events and/or changes in oceanic circulation patterns were recorded at Site 792. Recrystallized, poorly preserved, possibly reworked Eocene species (Globigerina senni and Globigerapsis sp.) were recorded in sediments at Site 793.
\end{abstract}

\section{INTRODUCTION}

On Leg 126 of the Ocean Drilling Program (ODP), the JOIDES Resolution drilled 19 holes in the northern part of the Izu-Bonin Arc. In Quaternary sequences, faunal abundance varies by a factor of $10^{5}-10^{6}$, reflecting mainly dilution by volcanogenic as well as terrigenous constituents, and therefore could not be used for paleotemperature reconstructions. The biostratigraphic usefulness of planktonic foraminifers in pre-Quaternary sequences was limited by rare occurrences and poor preservation within dissolution facies, by hiatuses, by extraneously deposited sediments, and by discontinuous coring. Because species ranges vary with region and/or author, whenever possible I used the northwest Pacific zonations (Keller, 1978, 1979a, 1979b, 1980a, 1980b, 1980c, 1981a, 1981b, 1983) except for intervals where dissolution or hiatuses were indicated.

\section{METHODS}

Approximately $10-15 \mathrm{~cm}^{3}$ of sediments were wet sieved through a 250 -mesh screen $(63 \mu \mathrm{m})$. Unconsolidated sediments were soaked in a $2 \% \mathrm{Na}_{2} \mathrm{CO}_{3}$ solution and further disaggregated in an ultrasonic bath cleaner for $20-30 \mathrm{~s}$. For consolidated sediments the following procedure was used: The sample was heated in an oven at $75^{\circ}-85^{\circ} \mathrm{C}$ for 8-10 days to eliminate the pore water, then cooled and covered with kerosene. After the kerosene had penetrated the pore space (about $24 \mathrm{hr}$ ), it was decanted and the sample covered with water. Considerable tension is produced in the pores when the water reacts with the kerosene, and the rock disaggregates. Hard shales, argillaceous sandstones, and limestones required further disaggregation: these samples were covered with a $30 \% \mathrm{H}_{2} \mathrm{O}_{2}$ solution to which Calgon was added and then heated. The last step had to be repeated several times for numerous samples. In Quaternary sequences, the coarse fraction, covering a $60-\mathrm{cm}^{2}$ tray, was examined in samples containing $>1000$ specimens. However, in numerous samples com-

'Taylor, B., Fujioka, K., et al., 1992. Proc. ODP, Sci. Results, 126: College Station, TX (Ocean Drilling Program). U.S.A.

${ }^{2}$ Department of Geology, Washington State University, Pullman, WA, 99164-2812, posed mainly of pumice or other nonbiogenic clasts, 15-20 trays were scanned for foraminiferal tests. Some of the nearly barren intervals contain few, poorly preserved, often unidentifiable specimens. Faunal abundance varied by a factor of $10^{5}-10^{6}$ and was not recorded because it reflects mainly dilution by volcanogenic as well as terrigenous constituents (Tables 1-5). Pre-Quaternary sequences contain corroded Neogene and Paleogene foraminifers.

\section{PLANKTONIC FORAMINIFERS}

All sites were drilled beneath the Kuroshio Current, which displaces isotherms northward. The foraminiferal assemblages in the study area belong to the Tropical Faunal Province (Bé, 1977).

In Tables 1-5, species are listed in order of increasing temperature of habitat: the lowest numbers corresponding to the coolest water or subsurface taxa. The distribution of several species is not unequivocally correlatable to temperatures. For example, Globigerina bulloides, Globigerina quinqueloba, Globoquadrina dutertrei, and Globigerinita glutinata have been found below both warm- and cold-water masses. According to Coulbourn et al. (1980), these species tend to follow concentrations of nutrients and may be opportunistic taxa, occupying environments unfavorable to other species. The distribution of Globorotalia inflata is also not related to temperature of habitat (Coulbourn et al., 1980). Compared to the composition of tropical assemblages in the overlying surface water (Bé, 1977), seafloor associations are enriched in solution-resistant forms such as Globorotalia inflata, Globoquadrina dutertrei, G. tumida, and G. menardii.

\section{Biostratigraphy}

\section{Quaternary}

The evolutionary appearance of Globorotalia truncatulinoides from its immediate ancestor Globorotalia tosaensis at the base of the Olduvai (1.88 Ma) magnetic event was proposed by Banner and Blow (1965) to mark the Pliocene/Pleistocene boundary. Published radiometric dates suggest that the Pliocene/Pleistocene boundary is $<2 \mathrm{Ma}$ (Berggren et al., 1985). Within the numerical zonal scheme of Blow (1969), this limit corresponds to the N21/N22 boundary. The range of dates in the equatorial north Pacific and the northwest Pacific for the first occurrence (FO) of G. truncatulinoides is ca. $1.65-1.88 \mathrm{Ma}$ 
(Thompson and Sciarrillo, 1978; Keller, 1978). In this study the FO of $G$. truncatulinoides defines the Zone N21/N22 limit, namely the Pliocene/Pleistocene boundary.

\section{Pre-Quaternary Zonation}

Planktonic foraminiferal zonation and placement of zonal boundaries was difficult, owing to hiatuses, extraneously deposited sediments, dissolution, facies, and discontinuous coring.

\section{Site 787}

Site 787 is located on the eastern edge of the Izu-Bonin forearc sedimentary basin, in the axis of Aoga Shima Canyon at $32^{\circ} 22.50^{\prime} \mathrm{N}$, $140^{\circ} 44.64 \mathrm{E}, 3259 \mathrm{~m}$ below sea level (mbsl) (Fig. 1) (Taylor, Fujioka, et al., 1990, pp. 63-96). Two holes were drilled at Site 787.

\section{Hole $787 B$}

Planktonic foraminiferal abundance at Site 787 reflects dilution by volcanogenic components, claystones, siltstones, and sandstones (Taylor, Fujioka, et al., 1990, pp. 63-96); their distributions in Hole 787B is shown in Table 1. The biostratigraphic usefulness of planktonic foraminifers in sedimentary sequences older than ca. $1.88 \mathrm{Ma}$ was limited by rare occurrences and the poor preservation of species.

Species distribution in Samples 126-787B-1R-CC, -2R-CC, and -3R-CC is shown in Table 1. Neither "pink" Globigerinoides ruber nor Globorotalia tosaensis were observed in these samples, suggesting that the age of Sample 126-787B-3R-CC is >0.2 Ma (Keller, 1979a; Thompson et al., 1979). The presence of Globorotalia truncatulinoides in Sample 126-787B-3R-CC indicates that the fauna was deposited < 1.88 Ma. A hiatus exists between Samples 126-787B-3R$\mathrm{CC}$ and $-4 \mathrm{R}-\mathrm{CC}, 1-3 \mathrm{~cm}$. Parts of Zones N21 and N20 are missing. Samples 126-787B-4R-CC, 1-3 cm, and -4R-CC are characterized by the presence of Globorotalia margaritae s (sinistral), G. crassaconica, G. conoidea, G. multicamerata d (dextral), G. tumida d $G$. cf. crassaformis, Globoquadrina venezuelana, G. altispira, Globigerina spp., Globigerinoides spp., and Sphaeroidinella dehiscens. They are assigned to Zones N18-N19. Globorotalia conoidea disappears in the upper part of Zones N19 in the northwest Pacific (Keller, 1979 b), ca. $3.5 \mathrm{Ma}$. The extinction of G. margaritae in northwest Pacific DSDP cores also occurred ca. 3.5 Ma (Keller, 1978, 1979a). It has been suggested that the last occurrence (LO) of this taxon in the north Pacific occurred ca. 4.40 Ma (COSOD II, 1987); this age should be viewed with caution as it does not support other results (see Keller, 1979a; Bolli and Saunders, 1985, and references therein; Kennett and Srinivasan, 1983, and references therein).

Another hiatus was recorded between Samples 126-787B-4R-CC and $-5 \mathrm{R}-\mathrm{CC}$; the latter sample contains specimens of Globorotalia plesiotumida (LO mid Zone N19), Globigerinoides obliquus extremus, and Globigerinoides conglobatus as well as numerous longranging taxa. The FO of these three species in DSDPnorthwest Pacific cores occurs ca. 7 Ma near the boundary between Zones N16/N17 (Keller, 1978, 1979b). Sample 126-787B-5R-CC was assigned to the lower part of Zone N17 (Berggren et al., 1985). Species that have their FO ca. 6 Ma during mid Zone N17, such as solution-resistant Pulleniatina primalis and Globoquadrina humerosa (Keller, 1980a), were not observed in this sample.

Only shell fragments were found in Samples 126-787B-6R-CC through -24R-CC. Sample 126-787B-25R-5, 54-56 cm, contains specimens of Globorotalia opima opima, G. opima nana, and Catapsydrax sp. and is assigned to Oligocene Zone P21. The occurrence of G. opima opima in DSDP northwest Pacific Core 292 ranges from $\sim 32$ to $\sim 28.2 \mathrm{Ma}$ (Keller et al., 1982; Keller and Barron, 1983). Sample 126-787B-30R-3, 93-95 cm, contains few specimens of $C$. dissimilis, G. opima nana, and Globigerina gortanii. The FO of the

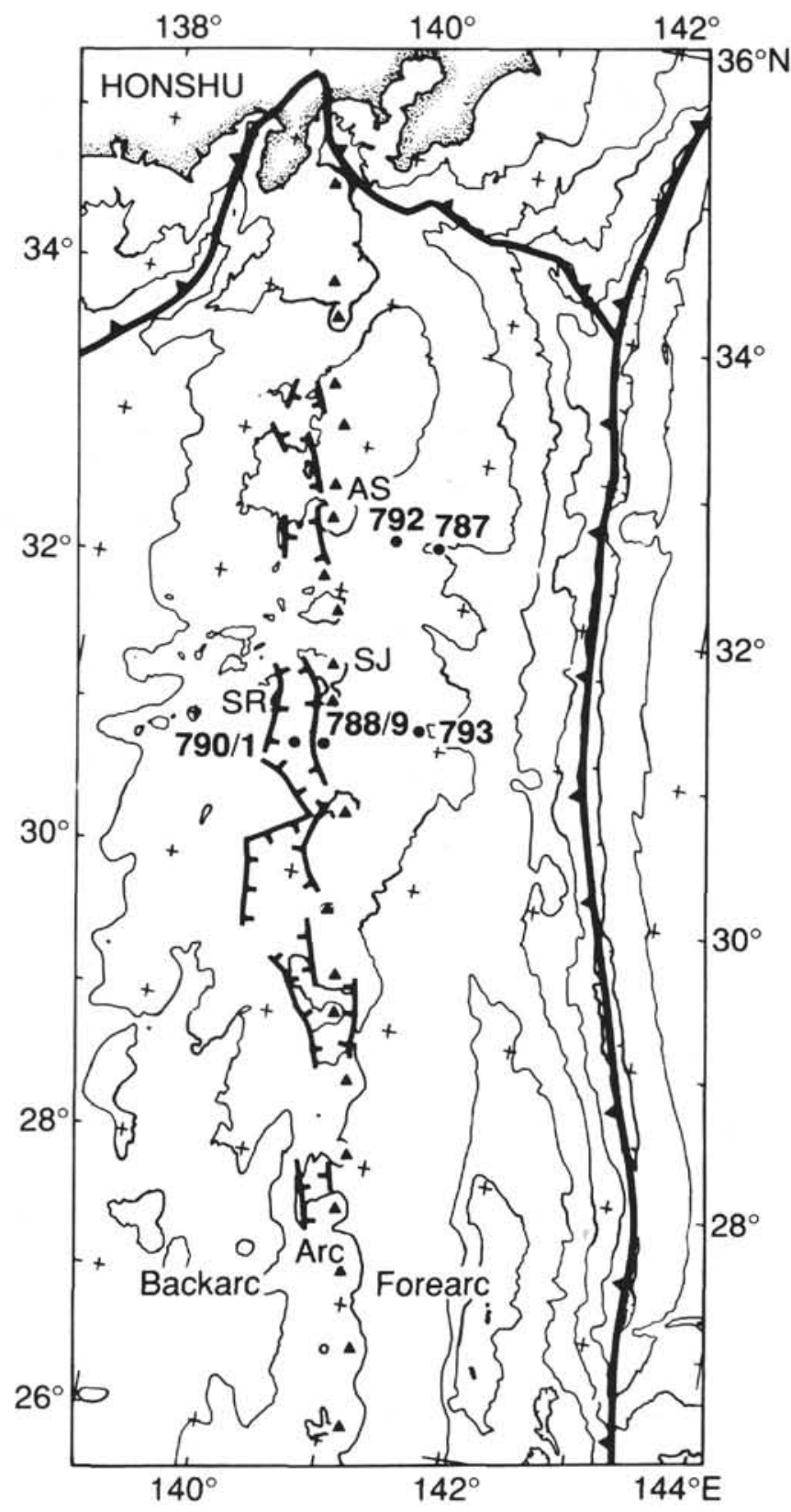

Figure 1. Bathymetric map of the Izu-Bonin intraoceanic island arc showing location of Leg 126 sites. SR = Susima Rift, SJ = Susima Jima, and AS = Aoga Shima (from Nishimura et al., 1991).

last species in northwest Pacific DSDP Site 292 was recorded in the Pseudohastigerina micra Zone (P19 and P18, ca. 37 Ma; Keller, 1982-1983). The LO of this species at the same site was placed in the middle of the Globigerina ampliapertura Zone (P20, ca. $31 \mathrm{Ma}$; Keller et al., 1982; Keller and Barron, 1983). A shorter stratigraphic range (FO $34 \mathrm{Ma}$ ) is given for this species in the generalized zonation table for the low-latitude Atlantic, Pacific, and Indian oceans (Keller et al., 1982, Keller and Barron, 1983).

\section{Site 788}

Site 788 is located on the eastern margin of the Sumisu Rift, at $30^{\circ} 55.36{ }^{\prime} \mathrm{N}, 140^{\circ} 00.21^{\prime} \mathrm{E}$ at $1102 \mathrm{mbsl}$ (Fig. 1) (Taylor, Fujioka, et al., 1990, pp. 97-126). Five holes were drilled at Site 788. 
Table 1. Distribution of planktonic foraminifers in selected samples from Hole 787B.

\begin{tabular}{|c|c|c|c|c|c|c|}
\hline & $\begin{array}{l}\text { Core, section, } \\
\text { interval }(\mathrm{cm})\end{array}$ & $\begin{array}{l}\text { Coiling } \\
\text { direction }\end{array}$ & IR-CC & $2 \mathrm{R}-\mathrm{CC}$ & $3 \mathrm{R}-\mathrm{CC}$ & $4 \mathrm{R}-\mathrm{CC}$ \\
\hline 1 & Globigerina pachyderma & $\mathbf{S}$ & & & & \\
\hline 2 & Globigerina pachyderma & D & $x$ & $x$ & $x$ & \\
\hline 3 & Globigerina cryophila & $\mathbf{S}$ & & & & \\
\hline $\begin{array}{l}4 \\
5\end{array}$ & $\begin{array}{l}\text { Globigerina cryophila } \\
\text { Globigerina hermanae }\end{array}$ & D & & & & \\
\hline 6 & Globigerina hermanae & D & & & & \\
\hline $\begin{array}{l}7 \\
8\end{array}$ & $\begin{array}{l}\text { Globigerina paraobesa } \\
\text { Globigerinita uvula }\end{array}$ & & & & & \\
\hline 9 & Globigerinita humilis & & $x$ & & & \\
\hline 10 & Globigerina bulloides. & & $\mathrm{x}$ & & $x$ & \\
\hline 11 & Globigerina falconensis & & & & & \\
\hline 12 & Globorotalia inflata & 3 chamb & $\mathbf{x}$ & $\mathrm{x}$ & $x$ & $\mathrm{x}$ \\
\hline 13 & Globorotalia inflata & 4 chamb & $x$ & $x$ & $x$ & $\mathbf{x}$ \\
\hline 14 & Globigerina quinqueloba & & $\mathrm{x}$ & $\mathrm{x}$ & & \\
\hline is & Globorotalia tosaensis & $S$ & & & & \\
\hline 16 & Globorotalia tosaensis & $\underset{S}{D}$ & & & & \\
\hline 17 & Globorotalia cf. tosaensis & $\mathrm{S}$ & & & & \\
\hline 18 & Globorotalia of. tosaensis & D & & & & $\mathbf{x}$ \\
\hline 19 & Globorotalia truncatulinoides & $\mathbf{S}$ & & $x$ & $x$ & $\mathrm{x}$ \\
\hline 20 & Globorotalia truncatulinoides & D & & & $\mathbf{x}$ & $\mathbf{x}$ \\
\hline 21 & Globorotalia scitula & & & $\mathrm{x}$ & & \\
\hline 22 & $\begin{array}{l}\text { Globorotalia scitula gigantea } \\
\text { Globorotalia pumilio }\end{array}$ & & & & & \\
\hline 24 & $\begin{array}{l}\text { Globorotalia pumilio } \\
\text { Globorotalia hirsuta }\end{array}$ & & & & & \\
\hline 25 & Globorotalia crassaformis & $\mathrm{x}$ & & & & $\mathrm{x}$ \\
\hline $\begin{array}{l}26 \\
27\end{array}$ & $\begin{array}{l}\text { Globorotalia sp. } 1 \\
\text { Globoquadrina conglomerata }\end{array}$ & & & & & \\
\hline 28 & $\begin{array}{l}\text { Globoquadrina conglomerata } \\
\text { Globorotalia sp. } 2\end{array}$ & & & & & \\
\hline 29 & Globoquadrina dutertrei & & $\mathrm{x}$ & $\mathrm{x}$ & $\mathrm{x}$ & \\
\hline 30 & Globigerinita glutinata & & $\mathrm{x}$ & $\mathbf{x}$ & $\mathrm{x}$ & \\
\hline 31 & Globigerinita iota & & & & & \\
\hline 32 & $\begin{array}{l}\text { Globigerinita sp. } \\
\text { Globorotalia pumilio }\end{array}$ & & & & & \\
\hline $\begin{array}{l}33 \\
34\end{array}$ & $\begin{array}{l}\text { Globorotalia pumilio } \\
\text { Globoquadrina pseudof oliata }\end{array}$ & & & & & \\
\hline 35 & Orbulina universa & & $x$ & & $x$ & $x$ \\
\hline 36 & Orbulina bilobata & & & & & \\
\hline 37 & Globigerina rubescens & & & & & \\
\hline 38 & Globigerinoides ruber & white & $\mathrm{x}$ & $\mathrm{x}$ & $\mathbf{x}$ & \\
\hline 39 & Globigerinoides ruber & pink & & & & \\
\hline $\begin{array}{l}40 \\
41\end{array}$ & & & $\mathrm{x}$ & $x$ & $x$ & \\
\hline 42 & $\begin{array}{l}\text { Globigerinella adamsi } \\
\text { Globigerinella aequilateralis }\end{array}$ & symm & & & $\mathbf{x}$ & \\
\hline 43 & Globigerinella aequilateralis & asymm & & & $x$ & \\
\hline 44 & Globigerina calida & & & & & \\
\hline $\begin{array}{l}45 \\
46\end{array}$ & $\begin{array}{l}\text { Hastigerina pelagi } \\
\text { Globigerina digitata }\end{array}$ & & & & & \\
\hline $\begin{array}{l}40 \\
47\end{array}$ & $\begin{array}{l}\text { Globigerina digitata } \\
\text { Globorotalia menardii }\end{array}$ & & $\mathrm{x}$ & $\mathrm{x}$ & & \\
\hline 48 & Globorotalia tumida & & & & $x$ & \\
\hline 49 & Globorotalia flexuosa & & & & & \\
\hline 50 & Pulleniatina obliquiloculata & & $\mathrm{x}$ & $\mathrm{x}$ & $\mathrm{x}$ & \\
\hline $\begin{array}{l}51 \\
52\end{array}$ & $\begin{array}{l}\text { Pulleniatina sp. } \\
\text { Sphaeroidinefla dehiscens }\end{array}$ & & & & & \\
\hline 53 & $\begin{array}{l}\text { Sphaerodinella dehiscens } \\
\text { Globorotalia anfracta }\end{array}$ & & & & & \\
\hline 54 & Globigerinoides sacculifer & & $x$ & $x$ & & \\
\hline 55 & Globigerinoides trilobus & & & $\mathrm{x}$ & $x$ & $x$ \\
\hline 56 & Globigerinoides tenellus & & & & & \\
\hline $\begin{array}{r}57 \\
58\end{array}$ & Globigerinoides conglobatus & & $x$ & & $\mathrm{x}$ & $\mathrm{x}$ \\
\hline $\begin{array}{l}58 \\
59\end{array}$ & $\begin{array}{l}\text { Candeina nitida } \\
\text { Globigerinoides sp. }\end{array}$ & & & & & \\
\hline 60 & $\begin{array}{l}\text { Globigerinoides sp. } \\
\text { Globoquadrina hexagona }\end{array}$ & & & & & \\
\hline
\end{tabular}

$\mathrm{D}=$ dextral, $\mathrm{S}=$ sinistral, chamb $=$ chambered, symm $=$ symmetrical, and asymm $=$ asymmetrical.

\section{Hole $788 \mathrm{C}$}

The following planktonic foraminifers were recovered in core catchers from Sample 126-788C-28X-CC and Samples 126-788D4R-CC, -5R-CC, and -6R-CC: Pulleniatina primalis (LO $3.5 \mathrm{Ma}$ ), Globigerina nepenthes (LO 3.5 Ma), Globorotalia puncticulata (FO 4.2-4.4 Ma at northwest Pacific DSDP Site 296; Keller, 1979b), G. crassaformis (FO 4.2-4.4 Ma; Keller 1979b), G. margaritae (LO 3.5-3.6 Ma at northwest Pacific DSDP Site 296, Keller 1979b). These samples were deposited between $4.2-4.4$ and ca. $3.5 \mathrm{Ma}$ in the early Pliocene, Zone N19 (Keller, 1978, 1979a, 1979b). The zonation of Bolli and Saunders (1985) was used almost exclusively during Leg 126. These authors, as well as others, place Zone N18 into the Pliocene. In this study, following Keller's zonation for the northwest Pacific (1978), I placed Zone N18 into the uppermost Miocene. All other samples analyzed from this hole were barren of planktonic foraminifers or contained unidentifiable shell fragments.

\section{Sites 790 and 791}

Site 790 is located at $30^{\circ} 54.96 \mathrm{~N}, 139^{\circ} 50.66^{\circ} \mathrm{E}$ at $2223 \mathrm{mbsl}$. Site $791,2.4 \mathrm{~km}$ away from Site 790 , is located at $30^{\circ} 54.97 \mathrm{~N}, 139^{\circ} 52.20^{\prime} \mathrm{E}$, at 2268 mbsl. Both sites are near the center of Sumisu Rift, a backarc graben, west of the active Izu-Bonin island-arc volcanoes (Fig. 1) (Taylor, Fujioka, et al., 1990c, pp. 127-220).
Five holes were drilled on the flat floor of the rift basin: three above a structural basement high at Site 790 and two at Site 791 near the center of the eastern half graben of the rift. Planktonic foraminiferal abundances at both sites primarily reflect the dilution of biogenic components by volcanogenic and terrigenous constituents and are not useful for paleoceanographic reconstructions; their distributions are listed in Tables 2 and 3.

\section{Holes 790A, 790B, and 790C}

The presence of Globorotalia truncatulinoides and Globigerinita iota (Keller, 1978) in Sample 126-790C-20X-3, 36-41 cm, indicates that the sediment at this level is $<1.88 \mathrm{Ma}$ (Table 2; Berggren et al., 1985). Another useful datum is the LO of Globorotalia tosaensis in Sample 126-790A-2H-CC; according to Keller (1979a), this species disappears in the northwest Pacific ca. 0.2 Ma (Table 2). No "pink" Globigerinoides ruber were observed at this site, suggesting that the core "top" has an age greater than $0.12 \mathrm{Ma}$, the datum for the disappearance of this taxon in the Pacific Ocean (Thompson et al., 1979). Because planktonic foraminifers are rare at many levels, the absence of "pink" G. ruber, a species constituting generally less than $1 \%$ of the total planktonic foraminifer population, may be an artifact of the low abundance of foraminifers (Table 2).

\section{Hole 791A and $791 B$}

The presence of $G$. truncatulinoides in Core 126-791B-47R-1, $41-43 \mathrm{~cm}$, indicates that the sediment is $<1.88 \mathrm{Ma}$ (Table 3 ). The LO of Globorotalia cf. tosaensis was observed in Core 126-791A-38XCC. A tentative age of $0.2 \mathrm{Ma}$ is suggested for this level. However, the stratigraphic range of G. tosaensis s.l. is uncertain. As in Site 790 cores, no "pink" G. ruber were observed at this site, suggesting that the true core "top" is missing. However, as in sediments at Site 790, volcanogenic components make up most of the coarse fraction in many examined samples; therefore, the absence of G. ruber "pink" may be an artifact of the low abundance of foraminifers. No additional datum planes suggested for other regions could be used at our sites. For example, Globorotalia flexuosa has a greater stratigraphic range in the Pacific than in the Atlantic Ocean (Ericson and Wollin, 1968; also see Site 792). Globigerina calida is present at Site 792 in the uppermost Pliocene. Therefore, its datum of FO at the base of N23 (Blow, 1969) does not apply to the northwest Pacific. Globigerinoides fistulosus, a solution-susceptible species, was either not present or was not preserved at our sites.

\section{Site 792}

Site 792 is located at $32^{\circ} 23.96{ }^{\circ} \mathrm{N}, 140^{\circ} 22.80^{\prime} \mathrm{E}$ at $1787 \mathrm{mbsl}$ in the western half of the Izu Bonin forearc sedimentary basin. Five holes were drilled at Site 792 (Taylor, Fujioka, et al., 1990, pp. 221-314). Hole 792A and the upper part of Hole 792B (Table 4) contain Quaternary planktonic foraminiferal sequences. Faunal abundance varies by a factor of $10^{5}-10^{6}$, reflecting mainly dilution by volcanogenic as well as by terrigenous constituents (Fig. 1); therefore, it could not be used for paleotemperature reconstructions. The lower section of Holes 792B, 792C, and 792D, and the upper four cores in Hole $792 \mathrm{E}$ contain Pliocene faunas. The lower portion of Hole 792E spans the Miocene through late Eocene. The fauna in Cores 126-792E-63R and $-64 R$, near the base of the hole, were deposited during late Eocene Zone P16 (38-37.3 Ma). The biostratigraphic usefulness of planktonic foraminifers in pre-Quaternary sequences was limited by rare occurrences and poor preservation within dissolution facies, by hiatuses, by extraneously deposited sediments, and by discontinuous coring. Because species ranges vary with region and/or author, whenever possible I used northwest Pacific zonations except for intervals where dissolution of hiatuses were indicated (Keller, 1978, 1979a, 1979b, 1980a, 1980b, 1980c, 1981a, 1981b, 1983). 
Table 2. Distribution of planktonic foraminifers in selected samples from Site $\mathbf{7 9 0 .}$

\begin{tabular}{|c|c|c|c|c|c|c|c|c|c|c|}
\hline & \multirow[b]{2}{*}{ Species } & \multirow[b]{2}{*}{$\begin{array}{l}\text { Coiling } \\
\text { direction }\end{array}$} & \multicolumn{8}{|c|}{$\begin{array}{c}\text { HOLE 790A } \\
\text { Core, section, interval }(\mathrm{cm})\end{array}$} \\
\hline & & & $\begin{array}{c}1 \mathrm{H}-1 \\
1-2\end{array}$ & $\begin{array}{c}1 \mathrm{H}-1 \\
2-4\end{array}$ & $\begin{array}{c}1 \mathrm{H}-4 \\
7-9\end{array}$ & $1 \mathrm{H}-\mathrm{CC}$ & $\begin{array}{c}2 \mathrm{H}-7 \\
44-46\end{array}$ & $2 \mathrm{H}-\mathrm{CC}$ & $\begin{array}{c}3 \mathrm{H}-5 \\
70-72\end{array}$ & $3 \mathrm{H}-\mathrm{CC}$ \\
\hline 1 & Globigerina pachvderma & $\mathrm{s}$ & & & & & & & & \\
\hline 2 & Globigerina pachyderma & $\mathrm{D}$ & $\mathrm{x}$ & $\mathrm{x}$ & $\mathrm{x}$ & $\mathrm{x}$ & & $\mathrm{x}$ & & \\
\hline $\begin{array}{l}3 \\
4\end{array}$ & $\begin{array}{l}\text { Globigerina cryophila } \\
\text { Globigerina cryophila }\end{array}$ & S & & & & $x$ & & $\mathrm{x}$ & & \\
\hline 5 & Globigerina hermanae & S & & & & & & & & \\
\hline 6 & $\begin{array}{l}\text { Globigerina hermanae } \\
\text { Globiering parobesa }\end{array}$ & D & & & & $\mathrm{x}$ & & & & \\
\hline 8 & Globigerinita uvula & & & & & & & $\mathrm{x}$ & & \\
\hline 9 & Globigerinita humilis & & $x$ & & & & & & & \\
\hline 10 & Globigerina bulloides & & & $\mathrm{x}$ & & $\mathrm{x}$ & & $\mathrm{x}$ & & $\mathrm{x}$ \\
\hline i1 & Globigerina falconensis & & $\mathrm{x}$ & $\mathrm{x}$ & & $\mathrm{x}$ & & $\mathrm{x}$ & & \\
\hline 12 & Globorotalia inflata & 3 chamb & $\mathrm{x}$ & $\mathrm{x}$ & $\mathrm{x}$ & $\mathrm{x}$ & & $\mathrm{x}$ & & \\
\hline 13 & Globorotalia inflata & 4 chamb & $\mathrm{x}$ & $\mathrm{x}$ & $\mathrm{x}$ & $\mathrm{x}$ & & $\mathrm{x}$ & & \\
\hline 14 & Globigerina quinqueloba & & $\mathrm{x}$ & $\mathrm{x}$ & & $\mathrm{x}$ & & $\mathrm{x}$ & & \\
\hline is & Globorotalia tosaensis & $\mathrm{s}$ & & & & & & & & \\
\hline 16 & Globorotalia tosaensis & D & & & & & & & & \\
\hline 17 & Globorotalia cf. tosaensis & S & & & & & & & & \\
\hline 18 & Globorotalia cf. tosaensis & D & & & & & & $\mathrm{x}$ & & \\
\hline 19 & Globorotalia truncatulinoides & s & & & & & & & & \\
\hline 20 & Globorotalia truncatulinoides & D & $\mathrm{x}$ & $\mathrm{x}$ & $\mathrm{x}$ & $\mathrm{x}$ & & & & \\
\hline 21 & Globorotalia scitula & & & $\mathrm{x}$ & & $\mathrm{x}$ & & & & \\
\hline 23 & $\begin{array}{l}\text { Globorotalia scitula gigantea } \\
\text { Globorotalia pumilio }\end{array}$ & & & & & & & & & \\
\hline 24 & Globorotalia hirsuta & & $\mathrm{x}$ & $\mathrm{x}$ & & & & & & \\
\hline 25 & Globorotalia crassaformis & & & & & & & & & \\
\hline 26 & Globorotalia sp. 1 & & & & & & $\mathrm{x}$ & & $\mathrm{x}$ & \\
\hline 27 & Globoquadrina conglomerata & & & & & & & & & \\
\hline 29 & $\begin{array}{l}\text { Globorotalia sp. } 2 \\
\text { Globoquadrina dutertrei }\end{array}$ & & $\mathrm{x}$ & $\mathrm{x}$ & $\mathrm{x}$ & x & & & & \\
\hline 30 & Globigerinita glutinata & & $\begin{array}{l}x \\
x\end{array}$ & $\begin{array}{l}x \\
x\end{array}$ & $\begin{array}{l}x \\
x\end{array}$ & $\begin{array}{l}\mathrm{x} \\
\mathrm{x}\end{array}$ & & & $\mathrm{x}$ & $\mathrm{x}$ \\
\hline 31 & Globigerinita iota & & $\hat{\mathrm{x}}$ & & & & & & & \\
\hline 32 & Globigerinita sp. & & & & & & & & & \\
\hline 33 & Globorotalia pumilio & & & & & & & & & \\
\hline 34 & $\begin{array}{l}\text { Globoquadrina pseudofoliata } \\
\text { Orbulina universa }\end{array}$ & & & $x$ & $\mathrm{x}$ & $x$ & & & & \\
\hline 36 & $\begin{array}{l}\text { Orbulina unversa } \\
\text { Orbulina bilobata }\end{array}$ & & & & & & & & & \\
\hline 37 & Globigerina rubescens & & & & & & & & & \\
\hline 38 & Globigerinoides ruber & white & $\mathrm{x}$ & $\mathrm{x}$ & $\mathrm{x}$ & $\mathrm{x}$ & & & & \\
\hline 39 & $\begin{array}{l}\text { Globigerinoides ruber } \\
\text { Globierinoides oomitulus }\end{array}$ & pink & & $x$ & & $x$ & & & & \\
\hline 41 & Globigerinella adamsi & & & $x$ & & & & & & \\
\hline 42 & Globigerinella aequilateralis & symm & $\mathrm{x}$ & $\mathrm{x}$ & $\mathrm{x}$ & $\mathrm{x}$ & & & & \\
\hline $\begin{array}{l}43 \\
44\end{array}$ & $\begin{array}{l}\text { Globigerinella aequilateralis } \\
\text { Globigerina calida }\end{array}$ & asymm & $x$ & $x$ & & & & & & \\
\hline 45 & Hastigerina pelagica & & & & & & & & & \\
\hline 46 & Globigerina digitata & & & & & & & & & \\
\hline 47 & Globorotalia menardii & & $\mathrm{x}$ & $\mathrm{x}$ & $\mathrm{x}$ & & & & & \\
\hline 48 & Globorotalia tumida & & $\hat{x}$ & $\hat{x}$ & & & & & & \\
\hline 49 & Globorotalia flexuosa & & & & & & & & & \\
\hline 50 & Pulleniatina obliquiloculata & & $\mathrm{x}$ & $\mathrm{x}$ & $\mathrm{x}$ & & & & & \\
\hline 51 & Pulleniatina sp. & & & & & & & & & \\
\hline 52 & Sphaeroidinella dehiscens & & $\mathrm{x}$ & $\mathrm{x}$ & & & & & & \\
\hline 53 & Globorotalia anfracta & & & & & & & & & \\
\hline 54 & Globigerinoides sacculifer & & $\mathrm{x}$ & $\mathrm{x}$ & $\mathrm{x}$ & & & & & \\
\hline 55 & Globigerinoides trilobus & & $\hat{x}$ & $\hat{\mathrm{x}}$ & & $\mathrm{x}$ & & & & \\
\hline 56 & Globigerinoides tenellus & & & & $\mathrm{x}$ & & & & & \\
\hline 57 & Globigerinoides conglobatus & & & $\mathrm{x}$ & $\hat{\mathrm{x}}$ & & & & & \\
\hline 58 & Candeina nitida & & & & & & & & & \\
\hline 59 & Globigerinoides sp. & & & & & & & & & \\
\hline 60 & Globoquadrina hexagona & & & & & & & & & \\
\hline
\end{tabular}

Notes: $\mathrm{D}=$ dextral, $\mathrm{S}=$ sinistral, chamb $=$ chambered, symm $=$ symmetrical, and asymm $=$ asymmetrical.

\section{Hole $792 A$}

At Hole 792A, the FO of G. truncatulinoides was observed in Sample 126-792A-10H-1, 70-72 cm, and in Sample 126-792B-5XCC (Table 4).

Other valuable zonal indicators are Globigerinita iota and Globigerinoides tenellus, which have their FOs also at the Pliocene/Pleistocene boundary (Core 126-792A-10H-1, 70-72 cm; Table 4; Keller, 1978; Parker, 1967). Several other useful datum planes are present at this site. The LO of Globoquadrina humerosa s.s. occurs in Sample 126792A-8H-1, 97-101 cm (Table 4). This datum level was dated ca. 1.49 $\mathrm{Ma}$ in Pacific Core RC11-220 (Bielak and Briskin, 1978). The LO of Globorotalia tosaensis occurs in Sample 126-792A-5H-4, 69-71 cm; according to Keller (1979a), this species disappears in the northwest Pacific ca. 0.2 Ma (Table 4). The LO of Globigerinoides ruber "pink" is in Section 126-792A-3H-CC. According to Thompson et al. (1979), the datum for this event is ca. $0.12 \mathrm{Ma}$ (Table 4).
There are no detectable paleoceanographic fluctuations in Quaternary sequences: Sample 126-792A-2H-CC contains a few, exclusively warm-water species, and the fauna of Sample 126-792A-4H-2, $70-72 \mathrm{~cm}$, is composed of cool-temperature taxa. In both samples volcanic ash constitutes almost the entire coarse fraction (Table 4), and the few specimens observed are insufficient for paleotemperature reconstruction. As discussed in a previous section, in all Quaternary samples the overprint of volcanic ash abundance variations over faunal fluctuations is so large that no paleoceanographic interpretations could be deduced.

\section{Hole $792 B$}

The presence of Globoquadrina venezuelana, Globoquadrina humerosa, and Globorotalia puncticulata places Sample 126-792B7X-CC in Pliocene Zone N21. The extinction of $G$. venezuelana was dated at 2.7 Ma in the tropical northwest Pacific (Keller, 1979a); the 
Table 2 (continued).

\begin{tabular}{|c|c|c|c|c|c|c|c|c|c|c|c|c|c|c|}
\hline \multicolumn{6}{|c|}{$\begin{array}{c}\text { HOLE 790B } \\
\text { Core, section, interval }(\mathrm{cm})\end{array}$} & \multicolumn{9}{|c|}{$\begin{array}{c}\text { HOLE 790C } \\
\text { Core, section, interval }(\mathrm{cm})\end{array}$} \\
\hline $2 \mathrm{H}-\mathrm{CC}$ & $3 \mathrm{H}-\mathrm{CC}$ & 7H-CC & $8 \mathrm{H}-\mathrm{CC}$ & $10 \mathrm{X}-\mathrm{CC}$ & $11 \mathrm{X}-\mathrm{CC}$ & 9H-CC & $\begin{array}{c}13 X-3 \\
104-106\end{array}$ & $14 \mathrm{X}-\mathrm{CC}$ & $15 \mathrm{X}-\mathrm{CC}$ & 17X-CC & $18 \mathrm{X}-\mathrm{CC}$ & $19 \mathrm{X}-\mathrm{CC}$ & $\begin{array}{l}20 X-3 \\
36-41\end{array}$ & $20 \mathrm{X}-\mathrm{CC}$ \\
\hline $\mathrm{x}$ & & $\mathrm{x}$ & & $\mathrm{x}$ & & & $x$ & & $\begin{array}{l}\mathrm{x} \\
\mathrm{x}\end{array}$ & & $x$ & $x$ & $x$ & $x$ \\
\hline & & & & & & & $x$ & & $x$ & & & & & \\
\hline & & $x$ & & & & & $\begin{array}{l}x \\
x\end{array}$ & & & & & & & \\
\hline $\begin{array}{l}x \\
x\end{array}$ & $\bar{x}$ & & & $x$ & & & $x$ & $\mathrm{x}$ & $x$ & & $x$ & & $x$ & \\
\hline & $\begin{array}{l}x \\
x\end{array}$ & $\mathrm{x}$ & & $\begin{array}{l}x \\
x\end{array}$ & $\mathrm{x}$ & & $\begin{array}{l}x \\
x\end{array}$ & $x$ & $\begin{array}{l}x \\
x\end{array}$ & $\mathrm{x}$ & $\mathrm{x}$ & $x$ & $\begin{array}{l}x \\
x\end{array}$ & $\mathrm{x}$ \\
\hline & $x$ & $x$ & & $\mathrm{x}$ & $x$ & & $\hat{x}$ & $\hat{\mathrm{x}}$ & $\hat{x}$ & $\mathrm{x}$ & $\mathrm{x}$ & $x$ & $x$ & $\mathrm{x}$ \\
\hline$x$ & $x$ & & & & & & $\bar{x}$ & & & & $x$ & $x$ & & $\mathrm{x}$ \\
\hline & & $\mathrm{x}$ & & $x$ & $x$ & & $x$ & & & & $\mathrm{x}$ & $x$ & $x$ & $\mathrm{x}$ \\
\hline $\mathrm{x}$ & $x$ & $\begin{array}{l}x \\
x\end{array}$ & & & & & $\begin{array}{l}x \\
x\end{array}$ & $x$ & $\mathrm{x}$ & $\mathrm{x}$ & $\begin{array}{l}x \\
x\end{array}$ & $\mathrm{x}$ & $\begin{array}{l}x \\
x\end{array}$ & $x$ \\
\hline & $\mathrm{x}$ & $\mathrm{x}$ & & $x$ & & & & & & & $\mathrm{x}$ & $x$ & & $\mathrm{x}$ \\
\hline$x$ & $\mathrm{x}$ & $x$ & & $\mathrm{x}$ & & & $x$ & & $x$ & $x$ & $\mathrm{x}$ & $\mathrm{x}$ & $x$ & $\mathrm{x}$ \\
\hline $\begin{array}{l}x \\
x\end{array}$ & $\begin{array}{l}x \\
x\end{array}$ & $x$ & & & $x$ & & $x$ & & $x$ & & $x$ & $x$ & $\begin{array}{l}x \\
x\end{array}$ & \\
\hline & & $x$ & & $x$ & $x$ & & $x$ & $x$ & & $x$ & $x$ & $\mathrm{x}$ & $\mathrm{x}$ & $x$ \\
\hline $\begin{array}{l}x \\
x\end{array}$ & $x$ & $\begin{array}{l}x \\
x\end{array}$ & & & & $x$ & $x$ & & $\mathrm{x}$ & & $\mathrm{x}$ & $x$ & $\mathrm{x}$ & $x$ \\
\hline$x$ & $x$ & $x$ & & $\mathrm{x}$ & & & $x$ & & & & $x$ & $x$ & $x$ & \\
\hline & & $\mathrm{x}$ & & & & $\mathrm{x}$ & & & & & & $x$ & & \\
\hline & & $\mathrm{x}$ & & $\begin{array}{l}x \\
x\end{array}$ & $x$ & & & & & $\mathrm{x}$ & & & & \\
\hline & $x$ & & & $\mathrm{x}$ & & & $x$ & & & & $\mathrm{x}$ & $x$ & $x$ & $x$ \\
\hline & & & & $x$ & & & $x$ & $\mathrm{x}$ & $x$ & & & $\mathrm{x}$ & $x$ & $x$ \\
\hline$x$ & $x$ & $x$ & & $\mathrm{x}$ & & & $\begin{array}{l}x \\
x\end{array}$ & $x$ & $\mathrm{x}$ & $x$ & $\mathrm{x}$ & $\begin{array}{l}x \\
x\end{array}$ & & \\
\hline & & & & & & & $x$ & & & & $x$ & $\begin{array}{l}x \\
x\end{array}$ & $\begin{array}{l}x \\
x\end{array}$ & $x$ \\
\hline$x$ & $x$ & & & $\mathrm{x}$ & & & & & & & $\mathbf{x}$ & $\begin{array}{l}x \\
x\end{array}$ & $\begin{array}{l}x \\
x\end{array}$ & $\mathrm{x}$ \\
\hline & & $x$ & & $\hat{x}$ & $x$ & $x$ & & $\mathrm{x}$ & & & & $\mathrm{x}$ & & $x$ \\
\hline & & $x$ & & $x$ & & & $\begin{array}{l}x \\
x\end{array}$ & $\mathrm{x}$ & & & $x$ & $x$ & $x$ & $x$ \\
\hline
\end{tabular}

minimum age of this sample is $2.7 \mathrm{Ma}$, suggesting a hiatus of at least 1 m.y. between Sections 126-792B-6X-CC and -7X-CC. Sample 126-792B-8X-2, 68-70 cm, contains, in addition to species mentioned in the previous sample, Sphaeroidinellopsis subdehiscens, placing the sample in Zone N19-N20 (LO Sphaeroidinellopsis spp. $3 \mathrm{Ma}$; Berggren et al., 1985). In addition to the species listed in the other Pliocene samples, Sample 126-792B-8X-4, 70-72 cm, contains specimens of Globigerina decoraperta and dextral G. multicamerata (FO N17, ca. 6 Ma; LO N20/N21, ca. 3 Ma; Bolli and Saunders, 1985; Kennett and Srinivasan, 1983) and assigned to Zones N19-N20. Sample 126-792B-8X-4-CC is composed mainly of volcanic glass; rare specimens of $G$. venezuelana, $G$. puncticulata, primitive $G$. inflata, Globigerinoides ruber "white," G. trilobus, and Globigerinita glutinata are present. The sample is assigned to Zones N19-N20. The presence of Globorotalia acostaensis limits the age of Sample 126-792B-9X-1, 70-72 cm, to Zones N19-N20 (Kennett and Srini- vasan, 1983). The faunal compositions of Cores 10 and 11 are similar to Core 126-792B-9X and are also assigned to Zones N19-N20.

\section{Holes $792 C$ and $792 D$}

Planktonic foraminiferal species composition in Sections 126792C-1X-CC and 126-792D-1X-CC is similar to that of Section 126-792B-9X-11-CC and is assigned to Zones N19-N20.

\section{Hole $792 E$}

The planktonic foraminifers in Cores 126-792E-1R through $-5 \mathrm{R}$ contain the same index taxa as those in Section 126-792B-9R-11-CC; they are assigned to Zones N19-N20. Sample 126-792E-6R-1, 58-60 $\mathrm{cm}$, is dominated by solution-resistant, robust specimens and is characterized by the presence of Globoquadrina altispira and Globorotalia 
Table 3. Distribution of planktonic foraminifers from selected samples from Site 791.

\begin{tabular}{|c|c|c|c|c|c|c|c|c|c|}
\hline & \multirow[b]{2}{*}{ Species } & \multirow[b]{2}{*}{$\begin{array}{c}\text { Coiling } \\
\text { direction }\end{array}$} & \multicolumn{7}{|c|}{$\begin{array}{c}\text { Hole 791A } \\
\text { Core, section, interval }(\mathrm{cm})\end{array}$} \\
\hline & & & $\begin{array}{l}4 \mathrm{H}-3 \\
60-62\end{array}$ & $\begin{array}{l}5 \mathrm{H}-3 \\
52-54\end{array}$ & $\begin{array}{l}16 \mathrm{H}-2 \\
95-97\end{array}$ & $31 \mathrm{X}-\mathrm{CC}$ & $38 \mathrm{X}-\mathrm{CC}$ & $41 \mathrm{X}-\mathrm{CC}$ & $46 \mathrm{X}-\mathrm{CC}$ \\
\hline & Globigerina pachyderma & s & & & & & & & \\
\hline$\frac{2}{3}$ & $\begin{array}{l}\text { Globigerina pachyderma } \\
\text { Globigerina cryophila }\end{array}$ & $\stackrel{\text { D }}{S}$ & $x$ & $\mathrm{x}$ & $x$ & $x$ & $x$ & $\mathrm{x}$ & $\mathrm{x}$ \\
\hline 4 & Globigerina cryophila & D & & $\mathrm{x}$ & $\mathrm{x}$ & & & $\mathrm{x}$ & $\mathrm{x}$ \\
\hline 5 & Globigerina hermanae & s & & & & & & & \\
\hline $\begin{array}{l}6 \\
7\end{array}$ & $\begin{array}{l}\text { Globigerina hermanae } \\
\text { Globigerina paraobesa }\end{array}$ & $\mathrm{D}$ & & & & & & & \\
\hline $\begin{array}{l}8 \\
9\end{array}$ & Globigerinita uvula & & $\mathrm{x}$ & & $x$ & & $x$ & & \\
\hline $\begin{array}{r}9 \\
10\end{array}$ & $\begin{array}{l}\text { Globigerinita humilis } \\
\text { Globigerina bulloides }\end{array}$ & & & $x$ & $x$ & & & $\mathrm{x}$ & \\
\hline 11 & Globigerina falconensis & & $x$ & $\begin{array}{l}x \\
x\end{array}$ & $x^{x}$ & $x$ & $x$ & $\begin{array}{l}x \\
x\end{array}$ & $\mathrm{x}$ \\
\hline 12 & Globorotalia inflata & 3 chamb & $\hat{\mathrm{x}}$ & $\hat{\mathrm{x}}$ & $\hat{\mathrm{x}}$ & $\hat{x}$ & $\hat{x}$ & $\hat{x}$ & $\hat{x}$ \\
\hline 13 & Globorotalia inflata & 4 chamb & $\hat{\mathrm{x}}$ & $\hat{\mathrm{x}}$ & $\hat{\mathrm{x}}$ & $\hat{\mathrm{x}}$ & $\hat{\mathrm{x}}$ & $\hat{x}$ & $\hat{\mathrm{x}}$ \\
\hline 14 & Globigerina quinqueloba & & $\mathrm{x}$ & $\mathrm{x}$ & $\mathrm{x}$ & & $\mathrm{x}$ & & \\
\hline 15 & Globorotalia tosaensis & $\mathrm{S}$ & & & & & & & \\
\hline 16 & $\begin{array}{l}\text { Globorotalia tosaensis } \\
\text { Globorotalia cf. tosaensis }\end{array}$ & $\stackrel{\mathrm{D}}{\mathrm{D}}$ & & & & & & & \\
\hline 18 & Globorotalia cf. tosaensis & $\mathrm{D}$ & & & & & $\mathrm{x}$ & & \\
\hline 19 & Globorotalia truncatulinoides & $\mathrm{s}$ & & & $\mathrm{x}$ & & & & \\
\hline 20 & Globorotalia truncatulinoides & D & $\mathrm{x}$ & $\mathrm{x}$ & & $\mathrm{x}$ & $\mathrm{x}$ & & $\mathrm{x}$ \\
\hline $\begin{array}{l}21 \\
22\end{array}$ & $\begin{array}{l}\text { Globorotalia scitula } \\
\text { Globorotalia scitula gigantea }\end{array}$ & & & $\mathrm{x}$ & & & $\mathrm{x}$ & & \\
\hline 23 & Globorotalia pumilio & & $\mathrm{x}$ & & & & $x$ & & \\
\hline 24 & Globorotalia hirsuta & & $\mathrm{x}$ & & & & & & \\
\hline $\begin{array}{l}25 \\
26\end{array}$ & & & & & & & & & \\
\hline 27 & $\begin{array}{l}\text { Globorotalia sp. } 1 \\
\text { Globoquadrina conglomerata }\end{array}$ & & & & & & & & \\
\hline 28 & Globorotalia sp. 2 & & & & & & & & \\
\hline 29 & Globoquadrina dutertrei & & $\mathrm{x}$ & $\mathrm{x}$ & $\mathrm{x}$ & $\mathrm{x}$ & $x$ & $\mathrm{x}$ & $\mathrm{x}$ \\
\hline 30 & Globigerinita glutinata & & $\mathrm{x}$ & $\mathrm{x}$ & $\mathrm{x}$ & & $\mathrm{x}$ & $\mathrm{x}$ & $\mathrm{x}$ \\
\hline 31 & Globigerinita iota & & & $\hat{\mathrm{x}}$ & & & & & \\
\hline 32 & Globigerinita sp. & & & & & & & & \\
\hline $\begin{array}{l}33 \\
34\end{array}$ & $\begin{array}{l}\text { Globorotalia pumilio } \\
\text { Globoquadrina pseudofoliata }\end{array}$ & & & & & & & & \\
\hline $\begin{array}{l}35 \\
36\end{array}$ & Orbulina universa & & $\mathrm{x}$ & $\mathrm{x}$ & $\mathrm{x}$ & & $\mathrm{x}$ & & $\mathrm{x}$ \\
\hline 37 & $\begin{array}{l}\text { Orbulina bilobata } \\
\text { Globigerina rubescens }\end{array}$ & & $x$ & $x$ & & & & & \\
\hline $\begin{array}{l}38 \\
39\end{array}$ & Globigerinoides ruber & white & $\mathrm{x}$ & $\mathrm{x}$ & $\mathrm{x}$ & $\mathrm{x}$ & $\mathrm{x}$ & $\mathrm{x}$ & $\mathrm{x}$ \\
\hline 40 & Globigerinoides gomitulus & & $x$ & $x$ & $x$ & $x$ & $x$ & & $x$ \\
\hline & Globigerinella adamsi & & & & & & & & \\
\hline 42 & Globigerinella aequilateralis & symm & $\mathrm{x}$ & $\mathrm{x}$ & $\mathrm{x}$ & & $\mathrm{x}$ & & \\
\hline $\begin{array}{l}43 \\
44\end{array}$ & $\begin{array}{l}\text { Globigerinella aequilateralis } \\
\text { Globigerina calida }\end{array}$ & asymm & $x$ & $x$ & $x$ & $\mathrm{x}$ & & & \\
\hline 45 & Hastigerina pelagi & & & $x$ & & & & & \\
\hline 46 & Globigerina digitata & & $\mathrm{x}$ & $\mathrm{x}$ & $\mathrm{x}$ & & & & \\
\hline 47 & Globorotalia menardii & & & $x$ & & $x$ & $\mathrm{x}$ & & \\
\hline 48 & Globorotalia tumida & & & $\mathrm{x}$ & & $\mathrm{x}$ & $\mathrm{x}$ & & \\
\hline 49 & Globorotalia flexuosa & & & $\mathrm{x}$ & & & & & \\
\hline 50 & $\begin{array}{l}\text { Pulleniatina obliquiloculata } \\
\text { Pylleniating }\end{array}$ & & $\mathrm{x}$ & $\mathrm{x}$ & $\mathrm{x}$ & $\mathrm{x}$ & $\mathrm{x}$ & $\mathrm{x}$ & $\mathrm{x}$ \\
\hline 52 & $\begin{array}{l}\text { Pulleniatina sp. } \\
\text { Sphaeroidinella dehiscens }\end{array}$ & & $\mathrm{x}$ & & & & & & \\
\hline 53 & Globorotalia anfracta & & $\mathrm{x}$ & & & & & & \\
\hline 54 & Globigerinoides sacculifer & & $\mathrm{x}$ & $\mathrm{x}$ & & & $\mathrm{x}$ & & \\
\hline 55 & Globigerinoides trilobus & & $\mathrm{x}$ & & & $\mathrm{x}$ & $\mathrm{x}$ & $\mathrm{x}$ & $\mathrm{x}$ \\
\hline 56 & Globigerinoides tenellus & & $\hat{x}$ & $\mathrm{x}$ & & & & & $\mathrm{x}$ \\
\hline 57 & Globigerinoides conglobatus & & $\mathrm{x}$ & $\mathrm{x}$ & & & & & \\
\hline 58 & Candeina nitida & & & & & & & & \\
\hline $\begin{array}{l}59 \\
60\end{array}$ & $\begin{array}{l}\text { Globigerinoides sp. } \\
\text { Globoquadrina hexagona }\end{array}$ & & & & & & & & \\
\hline
\end{tabular}

Notes: $\mathrm{D}=$ dextral, $\mathrm{S}=$ sinistral, chamb = chambered, symm = symmetrical, and asymm = asymmetrical.

conoidea. The FO of the latter species is within Zone N17 and the LO is in Zone N19 in northwest Pacific DSDP at Site 296 (Keller, 1980a). Samples 126-792E-7R-CC through -11R-CC are barren or contain rare fragments unidentifiable at $100 \times$ magnification. Sample 126-792E-12R-3, $65-67 \mathrm{~cm}$, with a few broken specimens of $G$. conoidea and Globorotalia miozea (FOca. $18 \mathrm{Ma}$, Zone N6, and LO6-7 Ma, Zone N17), is tentatively assigned to late Miocene Zone N17 (Jenkins, 1985). Globigerina nepenthes, G. miozea, and G. conoidea, in addition to Globorotalia miotumida (LO 4.8 Ma; Jenkins, 1985) and Globigerinoides kennetti (range 8.4-8 Ma; Keller, 1980b) and several long-ranging taxa, are present in Sample 126-792E-12R-CC; this sample is assigned to Zone N16.

One broken specimen of Globorotalia was present in Sample 126-792E-13R-1, 34-36 cm. Long-ranging Globigerinoides bollii is present in Sample 126-792E-13R-1, 52-54 cm. Planktonic foraminifers were not observed in Core 126-792E-14R. Section 126-792E$15 \mathrm{R}-\mathrm{CC}$ contains specimens of G. nepenthes, Sphaeroidinellopsis subdehiscens (FO ca. 12.9 Ma, LO ca. 3 Ma; Keller et al., 1982; Berggren et al., 1985; Barron et al., 1985) and other long-ranging, solution-resistant taxa. This sample is assigned to Zone N16. Above Section 126-792E-15R-CC there is a hiatus. The lower part of Zone N16 appears to be missing (10-8 Ma). Sample 126-792E-16R-CC is characterized by the presence of Globorotalia menardii sp. A, $G$. venezuelana, Globigerinoides obliquus, G. bollii, S. subdehiscens, $G$. bulloides, and $S$. seminulina and is assigned to Zone N15; it probably has an age $>10.5 \mathrm{Ma}$.

Sample 126-792E-17R-CC contains specimens of $S$. subdehiscens, G. bollii, G. menardii sp. A (range Zones N13-N18; Bolli and Saunders, 1985), and S. seminulina. This sample is likely to belong to Zone N15 and has an age >10.5 Ma. Sample 126-792E-18R-CC is barren of foraminifers, and Samples 126-792E-19R-CC and -20R-CC do not contain zonal marker species. In Sample 126-792E-23R-1, 59-60 cm, the presence of $G$. peripheroronda suggests that the minimum age of this 
Table 3 (continued).

\begin{tabular}{|c|c|c|c|c|c|c|c|c|c|}
\hline \multicolumn{10}{|c|}{$\begin{array}{c}\text { Hole 791B } \\
\text { Core, section, interval }(\mathrm{cm})\end{array}$} \\
\hline 13R-CC & 24R-CC & 30R-CC & 32R-CC & 37R-CC & 38R-CC & $39 \mathrm{R}-\mathrm{CC}$ & 45R-CC & 46R-CC & $\begin{array}{l}47 R-1 \\
41-43\end{array}$ \\
\hline \multirow[t]{5}{*}{$x$} & $x$ & & $x$ & $x$ & $\mathrm{x}$ & $x$ & $x$ & $x$ & $x$ \\
\hline & & & $\mathrm{x}$ & $x$ & $x$ & $x$ & & $\mathrm{x}$ & \\
\hline & & & $x$ & $x$ & $x$ & $x$ & $x$ & $x$ & \\
\hline & $\mathrm{x}$ & & & & & $\begin{array}{l}x \\
x\end{array}$ & & $\mathrm{x}$ & \\
\hline & $\mathrm{x}$ & & $x$ & $x$ & $x$ & $\mathrm{x}$ & $x$ & $\mathrm{x}$ & \\
\hline $\begin{array}{l}x \\
x\end{array}$ & $x$ & & & $x$ & & $x$ & & $x$ & $x$ \\
\hline $\begin{array}{l}x \\
x\end{array}$ & $\begin{array}{l}x \\
x\end{array}$ & $\begin{array}{l}x \\
x \\
x\end{array}$ & $\begin{array}{l}x \\
x\end{array}$ & $\begin{array}{l}x \\
x \\
x\end{array}$ & $\begin{array}{l}x \\
x\end{array}$ & $\begin{array}{l}x \\
x\end{array}$ & $\begin{array}{l}x \\
x\end{array}$ & $\begin{array}{l}x \\
x\end{array}$ & $\begin{array}{l}x \\
x\end{array}$ \\
\hline \multirow[t]{2}{*}{$\begin{array}{l}x \\
x\end{array}$} & $x$ & $x$ & $x$ & $x$ & $x$ & $\mathrm{x}$ & $x$ & $\begin{array}{l}x \\
x\end{array}$ & $\mathrm{x}$ \\
\hline & & & & $x$ & $\begin{array}{l}\mathrm{x} \\
\mathrm{x}\end{array}$ & & $x$ & $x$ & $\mathrm{x}$ \\
\hline \multirow[t]{2}{*}{$\mathrm{x}$} & $x$ & $x$ & $x$ & $\mathrm{x}$ & $\begin{array}{l}x \\
x\end{array}$ & $x$ & & & $x$ \\
\hline & & & & $x$ & $x$ & $x$ & $x$ & $x$ & $x$ \\
\hline & & & $x$ & $\mathrm{x}$ & $x$ & & $x$ & $x$ & \\
\hline $\mathrm{x}$ & $x$ & & $x$ & $\mathrm{x}$ & $x$ & $\mathrm{x}$ & $\mathrm{x}$ & $x$ & \\
\hline$x$ & $x$ & & & $x$ & $x$ & $x$ & & $x$ & $x$ \\
\hline$x$ & $\mathrm{x}$ & & $x$ & $x$ & $x$ & $x$ & $x$ & $x$ & $\mathrm{x}$ \\
\hline$x$ & $x$ & & & $x$ & $x$ & $x$ & $x$ & $x$ & $\mathrm{x}$ \\
\hline \multirow[t]{8}{*}{$x$} & $x$ & & & $x$ & & & $x$ & $\mathrm{x}$ & \\
\hline & & & & & $\mathrm{x}$ & & $x$ & & $\begin{array}{l}x \\
x\end{array}$ \\
\hline & $x$ & & $x$ & & $x$ & & $x$ & $x$ & $\mathrm{x}$ \\
\hline & & & & $\mathrm{x}$ & $x$ & $x$ & $x$ & $x$ & \\
\hline & & & & & $x$ & & $x$ & & $\mathrm{x}$ \\
\hline & & & & $\mathrm{x}$ & $x$ & & $\mathrm{x}$ & & \\
\hline & & & & $\mathrm{x}$ & $\mathrm{x}$ & $\begin{array}{l}x \\
x\end{array}$ & $\mathrm{x}$ & & \\
\hline & & & & & $\mathrm{x}$ & & & & \\
\hline
\end{tabular}

sample is 13.4 Ma, the datum for the LO of this taxon (Keller, 1980a). Another hiatus exists between Samples 126-792E-23R-1, 59-60 cm, and -24R-CC; parts of Zone N10, Zones N9 and N8, and Zone N7 are missing. Sample 126-792E-24R-CC contains G. continuosa (FO ca. 19 Ma; LO 9-11 Ma), C. stainforthi (LO 19 Ma; Keller, 1980a), and G. siakensis. The estimated age of this sample is ca. $19 \mathrm{Ma}$ (near the Zone N5/N6 boundary). Sample 126-792E-26R-CC contains $C$. stainforthi, $G$. trilobus, and G. venezuelana. In Sample 126-792E-27R-2, 68-70 cm, the following species were recorded: G. kugleri (FO N4/P22 boundary, ca. 23.7 Ma; Berggren et al., 1985; LO N4/N5 boundary, ca. 21.7 Ma; Keller, 1981a), G. praedehiscens, G. binaiensis (range Zones P22-N5), G. primordius (rangeZones P22-N5), G. ciperoensis (range middle Zone $\mathrm{P} 21$, ca. $30 \mathrm{Ma}$; Zone N4/N5, ca. $22 \mathrm{Ma}$ ), G. parawoodi (range Zones N5-N7), and G. praebulloides. This sample is tentatively assigned to Zones N4/N5; the age of this sample is ca. $22 \mathrm{Ma}$ (Berggren et al., 1985).

In Samples 126-792E-28R-1,77-79 cm, and -28R-CC, specimens of G. trilobus (FO N4), G. kugleri, and C. dissimilis are accompanied by other long-ranging taxa. These samples are assigned to lower
Miocene Zone N4 (ca. 23 Ma). Sample 126-792E-29R-CC records a hiatus of 1-2 Ma: Globigerina tripartita, Globigerina sellii, and other species known to become extinct near the Oligocene/Miocene boundary were not observed in this sample. This sample contains specimens of Globigerina opima nana and C. dissimilis; it was assigned to late Oligocene Zone N3/P22 (sensu Bolli and Saunders, 1985) or "N4" or P22 (sensu Berggren et al., 1985). Sample 126-792E-30R-3, 46-48 $\mathrm{cm}$, contains specimens of Chiloguembelina cubensis; the LO of this species was during Zone P21/P22 (according to Berggren et al., 1985) and during Zone P21 ca. $30 \mathrm{Ma}$ (according to Keller et al., 1982, and Keller and Barron, 1983) and the FO in Zones P16-P17, ca. 37-38 $\mathrm{Ma}$ (Keller et al., 1982; Keller and Barron, 1983). Samples 126-792E40R-3, 62-64 cm, and -40R-CC contain long-ranging species, some of which originate in the Eocene; however, no Eocene index taxa are present; therefore, these samples are tentatively assigned to the early Oligocene. The following species were recorded: Globigerina tripartita, G. opima nana (late Eocene-Oligocene), Catapsydrax unicavus (late Eocene-early Miocene), C. dissimilis (late Eocene-Oligocene), 
Table 4. Distribution of planktonic foraminifers in selected samples from Site 792.

\begin{tabular}{|c|c|c|c|c|c|c|c|c|c|c|c|c|c|c|c|c|c|}
\hline & \multirow[b]{2}{*}{ Species } & \multirow[b]{2}{*}{$\begin{array}{l}\text { Coiling } \\
\text { direction }\end{array}$} & \multicolumn{15}{|c|}{$\begin{array}{c}\text { Hole } 792 \mathrm{~A} \\
\text { Core, section, interval }(\mathrm{cm})\end{array}$} \\
\hline & & & $\begin{array}{c}1 \mathrm{H}-3 \\
70-72\end{array}$ & $\begin{array}{r}1 \mathrm{H}-5 \\
70-72\end{array}$ & IH-CC & $\begin{array}{c}2 \mathrm{H}-1 \\
22-24\end{array}$ & $\begin{array}{c}2 \mathrm{H}-3 \\
57-62\end{array}$ & $\begin{array}{r}2 \mathrm{H}-3 \\
70-72\end{array}$ & $2 \mathrm{H}-\mathrm{CC}$ & $\begin{array}{r}3 \mathrm{H}-2 \\
70-72\end{array}$ & $\begin{array}{r}3 \mathrm{H}-4 \\
70-72\end{array}$ & $3 \mathrm{H}-\mathrm{CC}$ & $\begin{array}{c}4 \mathrm{H}-2 \\
70-72\end{array}$ & $\begin{array}{c}4 \mathrm{H}-4 \\
70-72\end{array}$ & $4 \mathrm{H}-\mathrm{CC}$ & $\begin{array}{r}5 \mathrm{H}-2 \\
70-72\end{array}$ & $\begin{array}{r}5 \mathrm{H}-4 \\
69-71\end{array}$ \\
\hline \multirow{44}{*}{ 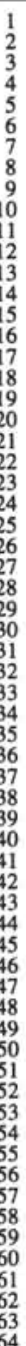 } & \multirow{44}{*}{ 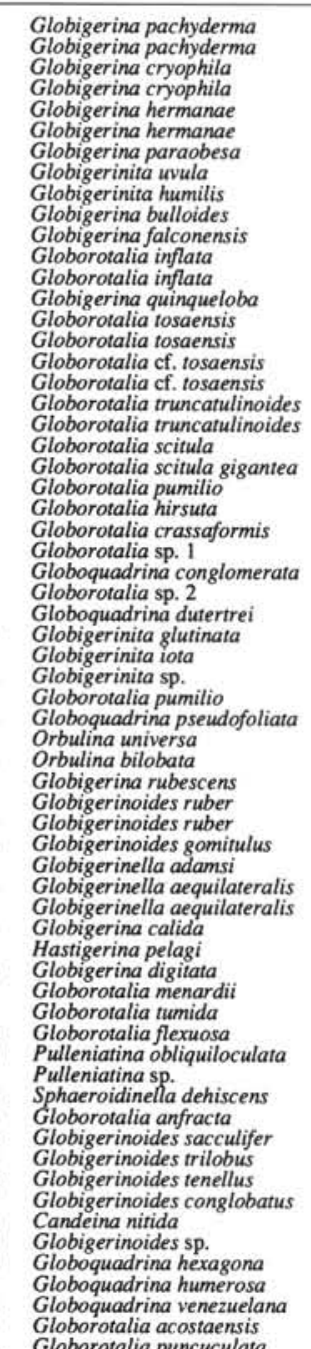 } & $\mathrm{s}$ & & & & & & & & & & & & & & & \\
\hline & & $\mathrm{D}$ & $x$ & $x$ & $x$ & $x$ & $x$ & $x$ & & $x$ & $x$ & $x$ & $x$ & $x$ & $x$ & $x$ & $x$ \\
\hline & & D & $\mathrm{x}$ & $x$ & $x$ & $\mathrm{x}$ & $x$ & $\mathrm{x}$ & & $\mathrm{x}$ & $x$ & & $x$ & $\mathrm{x}$ & $x$ & & \\
\hline & & D & & $x$ & $x$ & $\mathrm{x}$ & & & & $x$ & $x$ & & $x$ & $x$ & $x$ & $\mathrm{x}$ & $x$ \\
\hline & & & $\mathrm{x}$ & $x$ & $x$ & $x$ & & $x$ & & $x$ & $x$ & $x$ & & $x$ & $x$ & $\mathrm{x}$ & \\
\hline & & & $x$ & $x$ & $\begin{array}{l}x \\
x\end{array}$ & $x$ & $x$ & $x$ & & $\begin{array}{l}x \\
x\end{array}$ & $x$ & $\begin{array}{l}x \\
x\end{array}$ & & $\begin{array}{l}x \\
x\end{array}$ & $x$ & $\mathrm{x}$ & $x$ \\
\hline & & & $\hat{x}$ & $\hat{x}$ & $\hat{x}$ & $\hat{x}$ & $\hat{x}$ & $\hat{x}$ & & $\hat{x}$ & $\hat{x}$ & & & $\hat{x}$ & & & $\hat{x}$ \\
\hline & & 3 chamb & $\begin{array}{l}x \\
x\end{array}$ & $x$ & $\begin{array}{l}x \\
x\end{array}$ & $\begin{array}{l}x \\
x\end{array}$ & $\hat{x}$ & $\mathrm{x}$ & & $\hat{x}$ & $\hat{x}$ & $x$ & $x$ & $\hat{x}$ & $x$ & $x$ & $\hat{x}$ \\
\hline & & 4 chamb & $\hat{x}$ & $\hat{x}$ & $\hat{x}$ & $\hat{x}$ & $\hat{x}$ & $\mathrm{x}$ & & $\hat{x}$ & $\hat{x}$ & $\ddot{x}$ & $\hat{x}$ & $\hat{x}$ & $\hat{x}$ & $\hat{x}$ & $\hat{x}$ \\
\hline & & & $\hat{x}$ & $\hat{x}$ & $\hat{\mathrm{x}}$ & $\hat{x}$ & $\hat{x}$ & & & $\hat{x}$ & $\hat{x}$ & & & $\hat{x}$ & $\hat{x}$ & $\mathrm{x}$ & \\
\hline & & $\stackrel{\mathrm{S}}{\mathrm{D}}$ & & & & & & & & & & & & & & & $x$ \\
\hline & & $\stackrel{\mathrm{D}}{\mathrm{S}}$ & & & & & & & & & & & & & & & \\
\hline & & $\underset{\mathrm{S}}{\mathrm{D}}$ & $x$ & $x$ & & $x$ & & $x$ & & & & & & & & $x$ & $x$ \\
\hline & & $\mathrm{D}$ & $x$ & $\mathrm{x}$ & $\mathrm{x}$ & & $x$ & $x$ & & & $x$ & $x$ & & & $\mathrm{x}$ & $\mathrm{x}$ & $x$ \\
\hline & & & $\mathrm{x}$ & & $\hat{x}$ & & $\mathrm{x}$ & $\mathrm{x}$ & & $\mathrm{x}$ & $\mathrm{x}$ & $\mathrm{x}$ & & $\mathrm{x}$ & ; & & \\
\hline & & & $\begin{array}{l}x \\
x\end{array}$ & & & & & & & & $x$ & & & & $\mathrm{x}$ & & \\
\hline & & & & & $\mathrm{x}$ & $x$ & & $x$ & & $\mathrm{x}$ & $\hat{x}$ & & & $x$ & & & \\
\hline & & & & & $\mathrm{x}$ & $\mathrm{x}$ & $x$ & $x$ & & $\mathrm{x}$ & $\mathrm{x}$ & & & $x$ & $\mathrm{x}$ & $x$ & $x$ \\
\hline & & & & & & & & & & $x$ & $x$ & $x$ & & $x$ & $x$ & & $x$ \\
\hline & & & $\begin{array}{l}x \\
x\end{array}$ & $\begin{array}{l}x \\
x \\
x\end{array}$ & $x_{x}^{x}$ & $\begin{array}{l}x \\
x\end{array}$ & $\begin{array}{l}x \\
x\end{array}$ & $\begin{array}{l}x \\
x\end{array}$ & & $\begin{array}{l}x \\
x\end{array}$ & $\begin{array}{l}x \\
x\end{array}$ & $\begin{array}{l}x \\
x\end{array}$ & & $\begin{array}{l}x \\
x\end{array}$ & $\begin{array}{l}x \\
x\end{array}$ & $x$ & $\hat{x}$ \\
\hline & & & $\hat{x}$ & $\hat{\mathrm{x}}$ & $\hat{x}$ & $\hat{x}$ & $\hat{x}$ & & & $\hat{\mathrm{x}}$ & $\hat{x}$ & $\hat{\mathrm{x}}$ & & $\hat{\mathrm{x}}$ & $\hat{x}$ & $\hat{x}$ & $x$ \\
\hline & & & & & & & & & & & & & & & & & \\
\hline & & & & & & & & & & & & & & & & & \\
\hline & & & $\mathrm{x}$ & & $x$ & $x$ & $x$ & $\mathrm{x}$ & & & $\mathrm{x}$ & $x$ & & $x$ & $\mathrm{x}$ & $\mathrm{x}$ & $\mathrm{x}$ \\
\hline & & & $\mathrm{x}$ & & $\begin{array}{l}x \\
x\end{array}$ & $\mathrm{x}$ & $\mathrm{x}$ & $x$ & & $\mathrm{x}$ & $x$ & & & $\mathrm{x}$ & & & \\
\hline & & white & $\mathrm{x}$ & $\mathrm{x}$ & $x$ & $x$ & $x$ & $x$ & & $x$ & $x$ & $\mathrm{x}$ & & & $\mathrm{x}$ & $x$ & $x$ \\
\hline & & pink & & & $x$ & $x$ & $x$ & $x$ & & $\mathrm{x}$ & $x$ & $\begin{array}{l}x \\
x\end{array}$ & & $x$ & $x$ & & $x$ \\
\hline & & & $\mathrm{x}$ & & $\mathrm{x}$ & $\mathrm{x}$ & $x$ & $\mathrm{x}$ & & & $\mathrm{x}$ & $x$ & & $\mathrm{x}$ & $\mathrm{x}$ & $x$ & $x$ \\
\hline & & symm & $\mathrm{x}$ & & $\mathrm{x}$ & $\mathrm{x}$ & $x$ & $x$ & & $\mathrm{x}$ & $\mathrm{x}$ & $x$ & & & & & \\
\hline & & asymm & & $x$ & $\hat{x}$ & $\hat{x}$ & & & & & $x$ & $\mathrm{x}$ & & & $x$ & & \\
\hline & & & $x$ & $\hat{x}$ & $\hat{x}$ & $\hat{x}$ & $x$ & & & & $\mathrm{x}$ & & & $\mathrm{x}$ & & & \\
\hline & & & & & $x$ & & $x$ & & & & & & & $x$ & & & $x$ \\
\hline & & & $x$ & $x$ & $\begin{array}{l}x \\
x\end{array}$ & $x$ & $\begin{array}{l}x \\
x\end{array}$ & $x$ & & $\mathrm{x}$ & & $\mathrm{x}$ & & $\mathrm{x}$ & $\mathrm{x}$ & $\mathrm{x}$ & $\hat{x}$ \\
\hline & & & $\mathrm{x}$ & & $x$ & $x$ & $x$ & $x$ & & $\mathrm{x}$ & $x$ & $\begin{array}{l}x \\
x \\
x\end{array}$ & & $x$ & & $\mathrm{x}$ & \\
\hline & & & $\mathrm{x}$ & $x$ & $\begin{array}{l}x \\
x\end{array}$ & $\begin{array}{l}x \\
x\end{array}$ & $\mathrm{x}$ & $x$ & & $x$ & $x$ & $\begin{array}{l}x \\
x\end{array}$ & & $x$ & $x$ & $\mathrm{x}$ & $x$ \\
\hline & & & & & x & A & & & & & & & & $x$ & & & \\
\hline & & & $x$ & & $\begin{array}{l}x \\
x\end{array}$ & & & & & & & & & $x$ & & & \\
\hline & & & $\hat{\mathrm{x}}$ & $\mathrm{x}$ & $\hat{x}$ & $x$ & $x$ & & $x$ & $x$ & $x$ & & & $\mathrm{x}$ & & & $x$ \\
\hline & & & $\mathrm{x}$ & $x$ & $x$ & $x$ & $x$ & $x$ & $x$ & $x$ & $x$ & $x$ & & $x$ & $x$ & & $x$ \\
\hline & & & $x$ & $x$ & $x$ & $x$ & $x$ & $x$ & & $x$ & $x$ & $x$ & & $\begin{array}{l}x \\
x\end{array}$ & $x$ & & \\
\hline & & & $x$ & & $\mathrm{x}$ & $x$ & & & & & & & & & & & \\
\hline & & & & & $x$ & $\mathrm{x}$ & & & & $\mathrm{x}$ & & & & & $\mathrm{x}$ & & \\
\hline & & & & & & & & & & & & & & & & & \\
\hline & & & & & & & & & & & & & & & & & \\
\hline
\end{tabular}

Note: $\mathrm{D}=$ dextral, $\mathrm{S}=$ sinistral, chamb = chambered, symm = symmetrical, and asymm = asymmetrical.

G. venezuelana (middle Eocene-early Pliocene), and Globorotaloides suteri (late Eocene-Oligocene).

Samples 126-792E-41R-CC through -57R-1, 97-99 cm, are barren of planktonic foraminifers. In Sample 126-792E-57R-CC the following taxa were present: $C$. unicavus, C. dissimilis, and Globigerina angiporoides; they span the Eocene/Oligocene boundary. Samples 126-792E-63R-CC and -64R-1, 18-20 and 131-134 cm, contain specimens of Globorotalia cerroazulensis cocoaensis with rounded periphery (unkeeled), known to have become extinct before the end of the Eocene in Zone P16 (Toumarkine and Luterbacher, 1985; W. A. Berggren, pers. comm., 1991); Globigerina senni; and Globigerinatheka spp., which also became extinct before the end of the Eocene (Toumarkine and Luterbacher, 1985; W. A. Berggren, pers. comm., 1991). These Eocene index fossils are accompanied by long-ranging $C$. dissimilis, $G$. opima nana, G. angiporoides, and Globigerina cryptomphala. Samples exam- ined below this level were barren of planktonic foraminifers or contained unidentifiable species. The ages based on index planktonic foraminifers in Cores 126-792E-63R and -64R are much greater than the ages indicated by calcareous nannofossils (Taylor, Fujioka, et al., 1990, "Biostratigraphy" section, pp. 249-250).

\section{Site 793}

Site 793 is located at $31^{\circ} 06.33^{\circ} \mathrm{N}, 140^{\circ} 53.27^{\prime} \mathrm{E}$, at $2965 \mathrm{mbsl}$ in the center of the Izu-Bonin forearc sedimentary basin, west of the axis of the Izu-Bonin Trench and east of the volcanic front between Sumisu Jima and Tori Shima islands (Fig. 1) (Taylor, Fujioka, et al., 1990, pp. 315-403). Two holes were drilled at Site 793; Hole 793A contained Quaternary planktonic foraminifers (Table 5). Faunal abundance varies according to the extent of dilution of the foraminiferal tests by 


\begin{tabular}{|c|c|c|c|c|c|c|c|c|c|c|c|c|c|c|c|c|c|}
\hline \multicolumn{18}{|c|}{$\begin{array}{c}\text { Hole 792A } \\
\text { Core, section, interval }(\mathrm{cm})\end{array}$} \\
\hline $5 \mathrm{H}-4$ & $5 \mathrm{H}-5$ & $5 \mathrm{H}-6$ & & $6 \mathrm{H}-1$ & & $7 \mathrm{H}-1$ & $7 \mathrm{H}-2$ & $7 \mathrm{H}-3$ & & $8 \mathrm{H}-1$ & $8 \mathrm{H}-1$ & & $9 \mathrm{H}-2$ & $9 \mathrm{H}-4$ & & $10 \mathrm{H}-1$ & \\
\hline $91-93$ & $93-95$ & $86-88$ & $5 \mathrm{H}-\mathrm{CC}$ & $108-111$ & $6 \mathrm{H}-\mathrm{CC}$ & $59-61$ & $2-4$ & $2-4$ & 7H-CC & 93-97 & $97-101$ & $8 \mathrm{H}-\mathrm{CC}$ & $70-72$ & $36-38$ & $9 \mathrm{H}-\mathrm{CC}$ & $70-72$ & $10 \mathrm{H}-\mathrm{CC}$ \\
\hline \multirow[t]{2}{*}{$\mathrm{x}$} & $\mathrm{x}$ & $x$ & $x$ & $x$ & $\mathrm{x}$ & $x$ & $\mathrm{x}$ & $x$ & $x$ & $x$ & $x$ & $x$ & $x$ & $x$ & $x$ & $\mathrm{x}$ & $x$ \\
\hline & & & & $x$ & & & $\mathrm{x}$ & $\mathrm{x}$ & $x$ & & $x$ & & $x$ & $x$ & & & $x$ \\
\hline \multirow[t]{2}{*}{$x$} & $x$ & $\mathrm{x}$ & $x$ & $x$ & & & $x$ & $x$ & $\mathrm{x}$ & & $x$ & $\mathrm{x}$ & $x$ & & & & \\
\hline & $x$ & & & $\mathrm{x}$ & & & & & & & $\mathrm{x}$ & & $\mathrm{x}$ & & & & \\
\hline$x$ & & & & & & $x$ & & & & & & & & & & & \\
\hline $\bar{x}$ & $x$ & $x$ & & $\mathrm{x}$ & & & $x$ & $x$ & $x$ & $x$ & $\mathrm{x}$ & $x$ & $x$ & $x$ & $\mathrm{x}$ & $x$ & $\mathrm{x}$ \\
\hline $\mathrm{x}$ & $x$ & $x$ & & $x$ & & $x$ & & $x$ & $x$ & $x$ & $\mathrm{x}$ & & $x$ & & $\mathrm{x}$ & $x$ & $x$ \\
\hline$x$ & $x$ & $x$ & $x$ & $x$ & $x$ & $\mathrm{x}$ & $\mathrm{x}$ & $\mathrm{x}$ & $x$ & $x$ & $\mathrm{x}$ & $x$ & $x$ & $x$ & $x$ & $x$ & $x$ \\
\hline$x$ & $\mathrm{x}$ & $x$ & $\mathrm{x}$ & $\mathrm{x}$ & $x$ & $\vec{x}$ & $x$ & $\vec{x}$ & $\mathrm{x}$ & $\hat{x}$ & $\mathrm{x}$ & $x$ & $x$ & $x$ & $x$ & $x$ & $\mathrm{x}$ \\
\hline$x$ & $x$ & $x$ & & $x$ & & & $x$ & $\mathrm{x}$ & & & $\mathrm{x}$ & $\mathrm{x}$ & $\mathrm{x}$ & $x$ & $\mathrm{x}$ & & \\
\hline$x$ & $x$ & $x$ & $x$ & & & $x$ & & & & & & & $x$ & $x$ & $x$ & $x$ & $x$ \\
\hline \multirow[t]{2}{*}{$\mathrm{x}$} & $x$ & $x$ & $x$ & $x$ & & $x$ & & $x$ & & & & & $x$ & $x$ & $x$ & & $\mathrm{x}$ \\
\hline & & & & & $x$ & & & & & $\mathrm{x}$ & & & & & & & \\
\hline$x$ & $x$ & $x$ & $x$ & $\begin{array}{l}x \\
x\end{array}$ & $x$ & $x$ & & $x$ & & $\mathrm{x}$ & $x$ & $x$ & $\begin{array}{l}x \\
x\end{array}$ & $x$ & $\begin{array}{l}x \\
x\end{array}$ & $x$ & $x$ \\
\hline$x$ & & $\mathrm{x}$ & $x$ & & & & & $\mathrm{x}$ & & & $x$ & & $x$ & & & & \\
\hline \multirow[t]{2}{*}{$\mathrm{x}$} & $x$ & $x$ & $x$ & $x$ & $x$ & $x$ & $\mathrm{x}$ & $\mathrm{x}$ & $x$ & $x$ & $x$ & & $x$ & $x$ & $x$ & $x$ & $x$ \\
\hline & & & & & & & & & & & & & $x$ & & & & \\
\hline$x$ & $x$ & $x$ & $x$ & $x$ & $\mathrm{x}$ & $x$ & & $x$ & $\mathrm{x}$ & $x$ & $x$ & $x$ & $x$ & $x$ & $\mathrm{x}$ & $x$ & $x$ \\
\hline$x$ & $x$ & $x$ & $x$ & $x$ & & $\vec{x}$ & $x$ & $\hat{x}$ & & $\mathrm{x}$ & $\mathrm{x}$ & $\mathrm{x}$ & $\vec{x}$ & $x$ & $x$ & $x$ & $\mathrm{x}$ \\
\hline \multirow[t]{4}{*}{$\ddot{x}$} & $x$ & $x$ & & $\hat{x}$ & & $x$ & $\hat{x}$ & & & & $x$ & & $\hat{x}$ & $n$ & $\hat{n}$ & $\hat{x}$ & 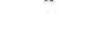 \\
\hline & $x$ & & & & & & & & & & & & & & & & \\
\hline & $x$ & & $x$ & $x$ & $x$ & & $x$ & $x$ & $x$ & $x$ & $\mathrm{x}$ & $x$ & $\mathrm{x}$ & $x$ & $\mathrm{x}$ & $x$ & $x$ \\
\hline & & & & & & & & $x$ & & & & & & & & & \\
\hline$x$ & $x$ & $\begin{array}{l}\mathrm{x} \\
\mathrm{x}\end{array}$ & $\begin{array}{l}\mathrm{x} \\
\mathrm{x}\end{array}$ & $\begin{array}{l}\mathrm{x} \\
\mathrm{x}\end{array}$ & $\mathrm{x}$ & $x$ & & $x$ & $x$ & $\mathrm{x}$ & $\mathrm{x}$ & $x$ & $x$ & $x$ & & $x$ & $x$ \\
\hline \multirow[t]{6}{*}{$x$} & $\mathrm{x}$ & $x$ & $\mathrm{x}$ & $\mathrm{x}$ & $x$ & $x$ & & $x$ & $\mathrm{x}$ & & $x$ & $x$ & $\mathrm{x}$ & $\mathrm{x}$ & & $x$ & \\
\hline & & & & & & & & & & & $x$ & & & & & & \\
\hline & $x$ & & & $x$ & & & & & $x$ & $x$ & $x$ & & & $x$ & $x$ & & \\
\hline & $\mathrm{x}$ & & & $x$ & & & & $x$ & $\bar{x}$ & & & $x$ & $x$ & $x$ & $x$ & & \\
\hline & & $x$ & & $x$ & $x$ & $x$ & & & & & & & $x$ & $x$ & $\mathrm{x}$ & $x$ & \\
\hline & $x$ & $\mathrm{x}$ & $x$ & $x$ & & & & $\mathrm{x}$ & & & $\mathrm{x}$ & $\mathrm{x}$ & $\mathrm{x}$ & $x$ & & $x$ & \\
\hline \multirow[t]{3}{*}{$x$} & $\mathrm{x}$ & $x$ & $x$ & $x$ & $x$ & & $\mathrm{x}$ & $\mathrm{x}$ & $x$ & $x$ & $x$ & & $x$ & $x$ & $x$ & $x$ & $x$ \\
\hline & $x$ & $\mathrm{x}$ & & $\bar{x}$ & $x$ & $x$ & $\bar{x}$ & $\mathrm{x}$ & & & $\mathrm{x}$ & & $x$ & $x$ & $\mathrm{x}$ & $x$ & $x$ \\
\hline & $x$ & & & $x$ & & & & & & & & & & & & $x$ & \\
\hline$x$ & $x$ & $\mathrm{x}$ & $x$ & $x$ & $x$ & $x$ & $\mathrm{x}$ & $x$ & $x$ & $x$ & $x$ & $\mathrm{x}$ & $x$ & $x$ & $x$ & $x$ & $x$ \\
\hline$x$ & $x$ & $x$ & $x$ & $x$ & $x$ & & $\mathrm{x}$ & & & & $\mathrm{x}$ & & $\mathrm{x}$ & $x$ & $\mathrm{x}$ & & $x$ \\
\hline \multirow[t]{2}{*}{$\hat{x}$} & & & & $x$ & & & & & & & & & $\mathrm{x}$ & & & & \\
\hline & $\mathbf{x}$ & & $x$ & $x$ & $\mathrm{x}$ & & $x$ & $x$ & $x$ & $x$ & & & $\mathrm{x}$ & $x$ & $x$ & & \\
\hline \multirow[t]{4}{*}{$x$} & $x$ & & & $x$ & $x$ & $x$ & & $x$ & $x$ & & $x$ & $x$ & $x$ & $\hat{x}$ & $\bar{x}$ & $x$ & $x$ \\
\hline & $x$ & $x$ & & $x$ & & $\ddot{x}$ & & $\mathrm{x}$ & & $\mathrm{x}$ & $\bar{x}$ & & $x$ & $x$ & $x$ & $x$ & \\
\hline & $x$ & $x$ & $x$ & $x$ & $x$ & & & $x$ & $x$ & $x$ & $\mathrm{x}$ & & $x$ & $x$ & & $x$ & $x$ \\
\hline & $x$ & & & & & $x$ & & & & & $x$ & & & & & & \\
\hline
\end{tabular}

nonbiogenic components, mainly volcanogenic elements. Hole 793B contains corroded Neogene and Paleogene foraminifers: their stratigraphic usefulness in pre-Quaternary sediments was limited by rare occurrences, hiatuses, dissolution facies, extraneously deposited sediments and discontinuous coring.

\section{Hole 793A}

The presence of Globigerinoides tenellus, and Globigerinita iota in Sample 126-793A-11H-CC places this sample in Zone N22. According to Parker (1967) and Keller (1978), the FO of these two species was recorded at the base of the Quaternary ca. $1.88 \mathrm{Ma}$. Another early Quaternary taxon, Globorotalia crassaformis hessi, is present in Sample 126-793A-10H-2, 102-104 cm, placing this sample in the early part of Zone N22 (Bolli and Saunders, 1985). The LO of Globorotalia tosaensis in Sample 126-793A-3H-4, 0-2 cm, suggests an age of $0.2 \mathrm{Ma}$ for this level (Keller, 1979a, 1979b). No "pink" Globigerinoides ruber were observed (LO 0.12 Ma; Thompson et al., 1979).
In many samples the total planktonic foraminifer abundance is very low because of dilution of the coarse fraction by nonbiogenic constituents. Globigerinoides ruber "pink" constitutes generally less than $1 \%$ of the foraminiferal population; its absence may be an artifact of the low abundance of foraminifers.

\section{Hole $793 B$}

Samples 126-793B-2R-1, 36-38 cm, and -2R-CC contain specimens of Globorotalia peripheroronda (FO at $16.9 \mathrm{Ma}$, Zone N8 in the northwest Pacific at DSDP Site 296 [Keller, 1980a], and LO at the same site in the middle of Zone N9, ca. 15 Ma [Keller, 1980a]). The other two species (Globorotalia praescitula and Sphaeroidinellopsis disjuncta) also have their LO in DSDPSite 296 in the middle of Zone N9. According to Keller (1980a), the fauna of Zones N8 and N9 were severely affected by dissolution; therefore, the total range of these species in the northwest Pacific DSDP cores is uncertain. In other regions, G. peripheroronda ranges from Zones N6 to N10, Globorotalia praescitula from Zones N6 
Table 4 (continued).

\begin{tabular}{|c|c|c|c|c|c|c|}
\hline & \multirow[b]{2}{*}{ Species } & \multirow[b]{2}{*}{$\begin{array}{l}\text { Coiling } \\
\text { direction }\end{array}$} & \multicolumn{4}{|c|}{$\begin{array}{c}\text { Hole 792B } \\
\text { Core, section, interval }(\mathrm{cm})\end{array}$} \\
\hline & & & $5 \mathrm{X}-\mathrm{CC}$ & $6 \mathrm{X}-\mathrm{CC}$ & $7 \mathrm{X}-\mathrm{CC}$ & $\begin{array}{l}8 X-2 \\
68-70\end{array}$ \\
\hline \multirow{7}{*}{$\begin{array}{l}1 \\
2 \\
3 \\
4 \\
5 \\
6 \\
7 \\
8 \\
9\end{array}$} & Globigerina pachyderma & $\mathbf{S}$ & & & & \\
\hline & $\begin{array}{l}\text { Globigerina pachyderma } \\
\text { Globigerina cryophila }\end{array}$ & $\stackrel{\mathrm{D}}{\mathrm{S}}$ & $\mathrm{x}$ & $x$ & $x$ & $\mathrm{x}$ \\
\hline & Globigerina cryophila & D & $\mathrm{x}$ & & & $x$ \\
\hline & Globigerina hermanae & $\mathrm{S}$ & & & & x \\
\hline & Globigerina hermanae & D & & & & \\
\hline & $\begin{array}{l}\text { Globigerinita uvula } \\
\text { Givesa }\end{array}$ & & & & & \\
\hline & Globigerinita humilis & & & & & \\
\hline \multirow{2}{*}{10} & Globigerina bulloides & & $\mathrm{x}$ & & & \\
\hline & Globigerina falconensis & & $\mathrm{x}$ & & & \\
\hline \multirow{3}{*}{$\begin{array}{l}12 \\
13 \\
14\end{array}$} & Globorotalia inflata & 3 chamb & $x$ & $x$ & $\mathrm{x}$ & $\mathrm{x}$ \\
\hline & Globorotalia inflata & 4 chamb & $\mathrm{x}$ & $\mathrm{x}$ & $\mathrm{x}$ & $\mathrm{x}$ \\
\hline & Globigerina quinqueloba & & & & $\mathrm{x}$ & $\mathrm{x}$ \\
\hline 15 & Globorotalia tosaensis & $\mathrm{s}$ & & & & \\
\hline \multirow{2}{*}{$\begin{array}{l}16 \\
17\end{array}$} & Globorotalia tosaensis & D & $\mathrm{x}$ & $x$ & & $x$ \\
\hline & Globorotalia cf. tosaensis & $\mathbf{s}$ & & & & \\
\hline \multirow{2}{*}{$\begin{array}{r}18 \\
19\end{array}$} & Globorotalia of. tosaensis & D & $x$ & & & \\
\hline & Globorotalia truncatulinoides & S & & & & \\
\hline \multirow{4}{*}{$\begin{array}{l}20 \\
21 \\
22 \\
23 \\
24\end{array}$} & Globorotalia truncatulinoides & D & $x$ & & & \\
\hline & Globorotalia scitula & & & $x$ & & \\
\hline & Globorotalia scitula gigantea & & & & & \\
\hline & $\begin{array}{l}\text { Globorotalia pumilio } \\
\text { Globorotalia hirsuta }\end{array}$ & & & & & \\
\hline \multirow{4}{*}{$\begin{array}{l}25 \\
26 \\
27 \\
28\end{array}$} & Globorotalia crassaformis & & $x$ & $\mathrm{x}$ & $\mathrm{x}$ & $x$ \\
\hline & Globorotalia sp. 1 & & & & & \\
\hline & $\begin{array}{l}\text { Globoquadrina conglomerata } \\
\text { Globorotalia sp. } 2\end{array}$ & & & & & $\mathrm{x}$ \\
\hline & Globoquadrina dutertrei & & $\mathrm{x}$ & $\mathrm{x}$ & & $x$ \\
\hline \multirow{5}{*}{$\begin{array}{l}29 \\
30 \\
31 \\
32 \\
33 \\
34\end{array}$} & Globigerinita glutinata & & & & $x$ & $\hat{x}$ \\
\hline & Globigerinita iota & & & & & \\
\hline & Globigerinita sp. & & & & & \\
\hline & Globorotalia pumilio & & & & & \\
\hline & Globoquadrina pseudofoliata & & & & & \\
\hline \multirow{3}{*}{$\begin{array}{l}35 \\
36 \\
37\end{array}$} & Orbulina universa & & $x$ & & $x$ & $\mathrm{x}$ \\
\hline & Orbulina bilobata & & & & & \\
\hline & Globigerina rubescens & & & & & \\
\hline \multirow{3}{*}{$\begin{array}{l}38 \\
39 \\
40\end{array}$} & Globigerinoides ruber & white & & $\mathbf{x}$ & $\mathrm{x}$ & $\mathbf{x}$ \\
\hline & Globigerinoides ruber & pink & & & & \\
\hline & Globigerinoides gomitulus & & $\mathrm{x}$ & & & \\
\hline \multirow{6}{*}{$\begin{array}{l}41 \\
42 \\
43 \\
44 \\
45\end{array}$} & Globigerinella adamsi & & & & & \\
\hline & Globigerinella aequilateralis & symm & & & & \\
\hline & Globigerinella aequilateralis & asymm & & & & \\
\hline & Globigerina calida & & & & & \\
\hline & Hastigerina pelagi & & & & & \\
\hline & Globigerina digitata & & & & & \\
\hline 47 & Globorotalia menardii & & $x$ & $x$ & $\mathrm{x}$ & $\mathrm{x}$ \\
\hline 48 & Globorotalia tumida & & $\mathrm{x}$ & $x$ & $\mathrm{x}$ & \\
\hline 49 & Globorotalia flexuosa & & & & & \\
\hline $\begin{array}{l}50 \\
51\end{array}$ & $\begin{array}{l}\text { Pulleniatina obliquiloculata } \\
\text { Pulleniatina sp. }\end{array}$ & & $x$ & $x$ & & \\
\hline 52 & Sphaeroidinella dehiscens & & $x$ & $x$ & $x$ & \\
\hline 53 & Globorotalia anfracta & & & & & \\
\hline 54 & Globigerinoides sacculifer & & & & & \\
\hline 55 & Globigerinoides trilobus & & $\mathrm{x}$ & $\mathrm{x}$ & $\mathrm{x}$ & \\
\hline 56 & Globigerinoides tenellus & & & & & \\
\hline 57 & Globigerinoides conglobatus & & $\mathrm{x}$ & & & \\
\hline 58 & Candeina nitida & & & & & \\
\hline 59 & Globigerinoides sp. & & & & & \\
\hline 60 & Globoquadrina hexagona & & & & & $\mathrm{x}$ \\
\hline 61 & Globoquadrina humerosa & & & & & \\
\hline 62 & Globoquadrina venezueiana & & & & $\mathrm{x}$ & \\
\hline 63 & Globorotallia acostaensis & & & & & \\
\hline 64 & Globorotalia puncuculata & & & & & \\
\hline
\end{tabular}

to N10, and S. disjuncta from Zones N9 to N17 (Bolli and Saunders, 1985; Barron et al., 1985). The tentative age of these samples is ca. 14.8-15.0 Ma (Zones N9-N10). In Samples 126-793B-8R-1, 60-62 cm, $-8 \mathrm{R}-\mathrm{CC},-9 \mathrm{R}-1,60-61 \mathrm{~cm},-9 \mathrm{R}-2,64-65 \mathrm{~cm}$, and $-9 \mathrm{R}-3,61-63 \mathrm{~cm}$, the following species were observed: $G$. peripheroronda, G. praescitula, Globigerinoides immaturus, and Globigerina connecta. The FO of the last taxon in DSDP Site 296 is at the Zone N4/5 boundary ca. $22 \mathrm{Ma}$ (Keller, 1980b); its LO is within a dissolution interval (Keller, 1980b). In other regions, its LO is at the Zone N7/8 boundary ca. $16.6 \mathrm{Ma}$ (Kennett and Srinivasan, 1983), suggesting an age of ca. 16.6 Ma for this sub-bottom depth.

In addition to the species listed in Core 126-793B-9R, the following taxa were recorded in Samples 126-793B-12R-2, 46-49 cm, and -12RCC: Globoquadrina altispira, Globorotalia pseudomiozea (FO mid Zone N6, ca. $20 \mathrm{Ma}$ ), Globorotalia semivera (LO top of Zone N8; Kennett and Srinivasan, 1983), Catapsydrax stainforthi (LO $16.7 \mathrm{Ma}$; Barron et al., 1985), and C. dissimilis (LO near the Zone N6 boundary in
DSDP Site 296; Keller, 1980a), suggesting an age of ca. 18.0 Ma for these samples. In Sample 126-793B-14R-2, 12-14 cm, the presence of Globigerina druryi (Zones N7-N15; Kennett and Srinivasan, 1983), G. pseudomiozea, G. connecta, C. dissimilis (LO N6/N7), and Globigerinoides trilobus immaturus (FO $22 \mathrm{Ma}$; Barron et al., 1985) suggest an age of ca. 18.0 Ma. In Samples 126-793B-16R-CC and -17R-4, 59-60 $\mathrm{cm}$, the presence of Globorotalia mendacis (LO $22.2 \mathrm{Ma}$ ), a minute globigerinoid, Chiloguembelina cubensis (range late Eocene-middle Oligocene; Keller et al., 1982; Keller and Barron, 1983), Globorotalia opima nana (late Eocene-early Miocene), G. continuosa (Oligoceneearly Miocene; Keller, 1980a), G. extans (FO Oligocene, LO $22.2 \mathrm{Ma}$; Keller, 1981b), G. cf. mayeri (FO late/middle Oligocene boundary, LO N14; Bolli and Saunders, 1985) suggests that these samples were deposited near the Oligocene/Miocene boundary ca. $24 \mathrm{Ma}$ (P22/N3-N4; Bolli and Saunders, 1985). The presence of $C$. cubensis may indicate reworking. No identifiable foraminifers were recorded between Cores 126793B-17R and -30R. The presence of $C$. cubensis (LO ca. $28 \mathrm{Ma}$ ), Globoquadrina venezuelana (FO middle Oligocene P20/N1 ca. $33 \mathrm{Ma}$; Bolli and Saunders, 1985), and Globigerina praebulloides suggests a middle Oligocene age of ca. 30 Ma for Sample 126-793B-31R-2, 93-95 $\mathrm{cm}$. In Sample 126-793B-52R-2, 33-35 cm, rare specimens of Globigerina ampliapertura (FO ca. $37 \mathrm{Ma}$, in Zone P17, late Eocene; LO ca. $33 \mathrm{Ma}$, in Zone $\mathrm{P} 19 / 20$ ) and G. pseudovenezuelana (FO ca. middle Eocene P11/12 boundary; LO ca. $34 \mathrm{Ma}$, Zone P18/P19) were recorded. Both species range from the Eocene to the early Oligocene (Keller et al., 1982; Keller and Barron, 1983; Bolli and Saunders, 1985; Toumarkine and Luterbacher, 1985). All samples examined below this level were barren of planktonic foraminifers or contained unidentifiable species. In Sample 126-793B-74R-1, 5-7 cm, several poorly preserved, recrystallized, Eocene species were identified by W. A. Berggren (Globigerina senni? and Globigerapsis sp.; pers. comm., 1991); they may be reworked. Other samples examined were barren of planktonic foraminifers or contained unidentifiable species.

\section{PALEOCEANOGRAPHY}

Present-day distribution patterns of seafloor assemblages reflect various water-mass parameters as well as shell preservation characteristics. Site 792 was drilled on the western half of the Izu-Bonin forearc sedimentary basin, beneath the Kuroshio Current, which displaces isotherms northward. The foraminifer assemblages in the study area belong to the Tropical Faunal Province (Bé, 1977).

In Quaternary sequences, faunal abundance varies by a factor of $10^{5}-10^{6}$ and reflects mainly dilution by volcanogenic as well as terrigenous constituents. It could not be used for paleotemperature reconstructions.

\section{Dissolution Effects}

Berger (1968, 1970, 1973, 1976) and Malmgren (1983, and references therein) have considered various factors that influence the dissolution of planktonic foraminifer tests, including (1) the degree of undersaturation of water with respect to $\mathrm{CaCO}_{3}$; (2) the rate of supply of calcareous vs. noncalcareous particles to the seafloor; (3) the length of time tests that are exposed to the cool corrosive bottom water; (4) rates of sedimentation; and (5) shell wall thickness, porosity, and microstructure. In our study the following criteria were used to establish the degree of dissolution: (1) percentage of preservation of solution-resistant species; (2) percentage of shell fragmentation; (3) wall texture (typically, test dissolution results in chalky texture, thinning, increase in pore size, and surface etchings); and (4) ratio of benthic/planktonic foraminifer tests, the former being more resistant to dissolution (Herman et al., 1989). Most pre-Pleistocene samples 
Table 5. Distribution of planktonic foraminifers in selected samples from Hole 793A.

\begin{tabular}{|c|c|c|c|c|c|c|c|c|c|c|c|c|c|c|}
\hline & \multirow[b]{2}{*}{ Species } & \multirow[b]{2}{*}{$\begin{array}{l}\text { Coiling } \\
\text { direction }\end{array}$} & \multirow[b]{2}{*}{$\begin{array}{l}1 \mathrm{H}- \\
1-2\end{array}$} & \multirow[b]{2}{*}{$\begin{array}{l}1 \mathrm{H}- \\
2-5\end{array}$} & \multirow[b]{2}{*}{$\begin{array}{c}1 \mathrm{H}-1 \\
6-8\end{array}$} & \multirow[b]{2}{*}{$\begin{array}{c}3 \mathrm{H}-4 \\
0-2\end{array}$} & \multicolumn{5}{|c|}{$\begin{array}{c}\text { Hole 793A } \\
\text { Core, section, interval }(\mathrm{cm})\end{array}$} & \\
\hline & & & & & & & $4 \mathrm{H}-\mathrm{CC}$ & $\begin{array}{c}5 \mathrm{H}-3 \\
2-4\end{array}$ & SH-CC & $\begin{array}{l}7 \mathrm{H}-3 \\
60-65\end{array}$ & $7 \mathrm{H}-\mathrm{CC}$ & $9 \mathrm{H}-\mathrm{CC}$ & $\begin{array}{c}10 \mathrm{H}-2 \\
102-104\end{array}$ & $11 \mathrm{H}-\mathrm{CC}$ \\
\hline 1 & Globigerina pachyderma & $\mathrm{S}$ & & & $\mathrm{x}$ & & & & & & & - & & \\
\hline 2 & $\begin{array}{l}\text { Globigerina pachyderma } \\
\text { Globigerina cryophila }\end{array}$ & D & & & $\hat{x}$ & & $x$ & $\mathrm{x}$ & & $x$ & & $x$ & & $\mathrm{x}$ \\
\hline 4 & Globigerina cryophila & $\mathrm{D}$ & & & & & $x$ & & & & & $x$ & & $\mathrm{x}$ \\
\hline 5 & Globigerina hermanae & $\mathrm{S}$ & & & & & $\lambda$ & & & & & & & \\
\hline 6 & $\begin{array}{l}\text { Globigerina hermanae } \\
\text { Globigerina paraobesa }\end{array}$ & $\overrightarrow{\mathrm{D}}$ & & & & & & & & & & & & \\
\hline 8 & Globigerinita uvula & & & & & & & & & $\mathrm{x}$ & $\mathrm{x}$ & & & \\
\hline 9 & Globigerinita humilis & & & & & & & & & x & $\boldsymbol{A}$ & & & \\
\hline 10 & Globigerina bulloides & & & & & & & & & $\mathrm{x}$ & & & $\mathrm{x}$ & \\
\hline 11 & Globigerina falconensis & & & & & & & & & $\hat{x}$ & & $\mathrm{x}$ & & \\
\hline 12 & Globorotalia inflata & 3 chamb & & & $\mathrm{x}$ & $\mathrm{x}$ & $\mathrm{x}$ & $\mathrm{x}$ & $\mathrm{x}$ & $\hat{x}$ & $\mathrm{x}$ & $\hat{x}$ & $\mathrm{x}$ & $\mathbf{x}$ \\
\hline 13 & Globorotalia inflata & 4 chamb & & & $\hat{x}$ & $\mathrm{x}$ & $\mathrm{x}$ & $\mathrm{x}$ & $x$ & $\mathrm{x}$ & $\mathrm{x}$ & $\mathrm{x}$ & $\mathrm{x}$ & $\mathrm{x}$ \\
\hline 14 & Globigerina quinqueloba & & $\mathrm{x}$ & & $\hat{x}$ & & $\hat{x}$ & & & & & & & \\
\hline 15 & Globorotalia tosaensis & $S$ & $\lambda$ & & $x$ & & $\mathrm{x}$ & & & & & & & \\
\hline 16 & Globorotalia tosaensis & D & & & & $\mathrm{x}$ & $x$ & $x$ & $x$ & $x$ & & $\mathrm{x}$ & $\mathrm{x}$ & \\
\hline 17 & Globorotalia cf. tosaensis & $\mathrm{S}$ & & & & & & & & & & & & \\
\hline 18 & Globorotalia cf. tosaensis & $\mathrm{D}$ & & & & & & $\mathrm{x}$ & & $\mathrm{x}$ & & & $\mathrm{x}$ & \\
\hline 19 & Globorotalia truncatulinoides & $\mathrm{S}$ & & & & & & & & & & & & \\
\hline 20 & Globorotalia truncatulinoides & D & & & & $\mathrm{x}$ & $\mathrm{x}$ & & & & & & & \\
\hline 21 & Globorotalia scitula & & & & & & $\mathrm{x}$ & & $x$ & $x$ & & & & \\
\hline 22 & $\begin{array}{l}\text { Globorotalia scitula gigantea } \\
\text { Globorotalia pumilio }\end{array}$ & & & & & & & $\mathrm{x}$ & & & & & & \\
\hline 24 & Globorotalia hirsuta & & & & & & & $\mathrm{x}$ & & & & & & \\
\hline 25 & Globorotalia crassaformis & & & & & & $\mathrm{x}$ & $\mathrm{x}$ & $\mathrm{x}$ & & & $\mathrm{x}$ & $\mathrm{x}$ & \\
\hline 26 & Globorotalia sp. 1 & & & & & & & & & & & & $\hat{x}$ & \\
\hline $\begin{array}{l}27 \\
28\end{array}$ & Globoquadrina conglomerata & & & & & & & & & & & & & \\
\hline 29 & Globoquadrina dutertrei & & & & & & $\mathrm{x}$ & $x$ & $\mathrm{x}$ & $x$ & $x$ & $\mathrm{x}$ & & \\
\hline 30 & Globigerinita glutinata & & & & $\mathrm{x}$ & & $\begin{array}{l}x \\
x\end{array}$ & $x$ & $x$ & $\begin{array}{l}x \\
x\end{array}$ & $\hat{x}$ & $\hat{x}$ & & \\
\hline 31 & Globigerinita iota & & & & $\mathrm{A}$ & & & & & & $\hat{x}$ & & & $\mathrm{x}$ \\
\hline 32 & Globigerinita sp. & & & & & & & & & & & & & \\
\hline 33 & Globorotalia pumilio & & & & & & & & & & & & & \\
\hline 34 & Globoquadrina pseudofoliata & & & & & & & & & & & & & \\
\hline 35 & Orbulina universa & & & & $x$ & & $\mathrm{x}$ & $x$ & $x$ & & & $x$ & $x$ & \\
\hline 36 & Orbulina bilobata & & & & & & & & & & & & & \\
\hline 37 & Globigerina rubescens & & & & & & & & & & & & & \\
\hline 38 & Globigerinoides ruber & white & & & $x$ & & $\mathrm{x}$ & $\mathrm{x}$ & $x$ & $\mathrm{x}$ & $x$ & $x$ & $x$ & $x$ \\
\hline 39 & Globigerinoides ruber & pink & & & & & & & & & & & & \\
\hline 40 & Globigerinoides gomitulus & & & & & & & $x$ & $x$ & $x$ & & $x$ & & \\
\hline 41 & $\begin{array}{l}\text { Globigerinella adamsi } \\
\text { Globigerinella aequilateralis }\end{array}$ & symm & & & & & $x$ & & $\times$ & $x$ & & & & \\
\hline 43 & $\begin{array}{l}\text { Globigerinella aequilateralis } \\
\text { Globigerinella aequilateralis }\end{array}$ & asymm & & & & & & & $\mathrm{x}$ & & & & & \\
\hline 44 & Globigerina calida & & & & & & & & & & $x$ & & & \\
\hline 45 & Hastigerina pelagi & & & & & & & & & & & & & \\
\hline 46 & Globigerina digitata & & & & & & & & & $\mathrm{x}$ & & & & \\
\hline 47 & Globorotalia menardii & & & & & $x$ & & $x$ & & $\hat{x}$ & & & & \\
\hline 48 & Globorotalia tumida & & & & & $x$ & $\mathrm{x}$ & $\mathrm{x}$ & & $x$ & & $\mathrm{x}$ & & \\
\hline 49 & Globorotalia flexuosa & & & & & & & & & & & & & \\
\hline 50 & Pulleniatina obliquiloculata & & & & & & $\mathrm{x}$ & & $\mathrm{x}$ & $\mathrm{x}$ & & $x$ & & \\
\hline 52 & Sphaeroidinella dehiscens & & & & & & & $\mathrm{x}$ & $\mathrm{x}$ & $x$ & $\mathrm{x}$ & & & \\
\hline 53 & Globorotalia anfracta & & & & & & & & & & & & & \\
\hline 54 & Globigerinoides sacculifer & & & & & & & & $\mathrm{x}$ & $\mathrm{x}$ & & & & \\
\hline 55 & Globigerinoides trilobus & & & & $\mathrm{x}$ & & $\mathrm{x}$ & $\mathrm{x}$ & $x$ & $\mathrm{x}$ & & $\mathrm{x}$ & & \\
\hline 56 & Globigerinoides tenellus & & & & $\hat{x}$ & & & & $\hat{x}$ & $\hat{x}$ & & & & $\mathrm{x}$ \\
\hline 57 & Globigerinoides conglobatus & & & & & & $\mathrm{x}$ & & $\hat{x}$ & & & & & \\
\hline 58 & Candeina nitida & & & & & & & & & & & & & \\
\hline 59 & Globigerinoides sp. & & & & & & & & & & & & & \\
\hline 60 & Globoquadrina hexagona & & & & & & & & $\mathrm{x}$ & & & & & \\
\hline
\end{tabular}

Notes: $\mathrm{D}=$ dextral, $\mathrm{S}=$ sinistral, chamb $=$ chambered, symm $=$ symmetrical, and asymm $=$ asymmetrical.

have been affected by dissolution; the ranking has been published by Taylor, Fujioka, et al. (1990, pp. 671-786).

Changes in the intensity of cold Antarctic bottom water were probably the major cause for the dissolution of calcareous sediments and occurrence of hiatuses. Varying sedimentation rates and fluctuations in the $\mathrm{CCD}$ have also contributed to the dissolution of planktonic foraminifer shells. At Site 792 the first hiatus was detected near the Pliocene/Pleistocene boundary in Hole 792B between Cores 126792B-6X and -7X ( 98.2 m below seafloor [mbsf]; Fig. 2) and is at least $1 \mathrm{~m} . \mathrm{y}$. in duration. This hiatus marks one of the major late Neogene global cold events, as indicated by various oxygen isotope records (e.g., Shackleton and Kennett, 1975; Blanc, 1981; Williams et al., 1985), and is referred to as Cold Event 1 in this chapter. A dissolution interval preceded by a hiatus was observed below Core 126-792E-6R ( 183 mbsf; Fig. 2); only solution-resistant taxa were preserved. The dissolution event occurred roughly around 3-3.4 Ma and is referred to as Cold Event 2 (Fig. 2). This cold pulse was preceded by a period of nearly complete dissolution of planktonic foraminiferal shells between Cores 126792E-7R and 11R ( 193-240 mbsf), ca. 6 to 3-3.4 Ma (Cold Event 3).

Between ca. 8 and $10 \mathrm{Ma}$ another hiatus was detected. This time interval represents another cold episode, Cold Event 4 (Cores 126-792E$14 \mathrm{R}$ and $-15 \mathrm{R}, \sim 270 \mathrm{mbsf}$ ) and correlates temporarily with cold intervals in equatorial Pacific isotopic records (e.g., Pisias et al., 1985). The period of dissolution between ca. 11 and $13 \mathrm{Ma}$ (Cores 126-792E-18R through $-22 \mathrm{R}, \sim 300-347 \mathrm{mbsf}$ ) is preceded by a hiatus between ca. 13.4 and ca. $19 \mathrm{Ma}$ (between Cores 126-792E-23R and -24R, 347-367 mbsf). The hiatus dissolution episode (Cold Event 5) is equated with the major middle Miocene cold period, which has been interpreted as an indication of a large-scale buildup in the Antarctic ice sheet or a combination of a major cooling of oceanic deep-water masses and a significant ice sheet growth (Pisias et al., 1985, and references therein). A hiatus between 23 

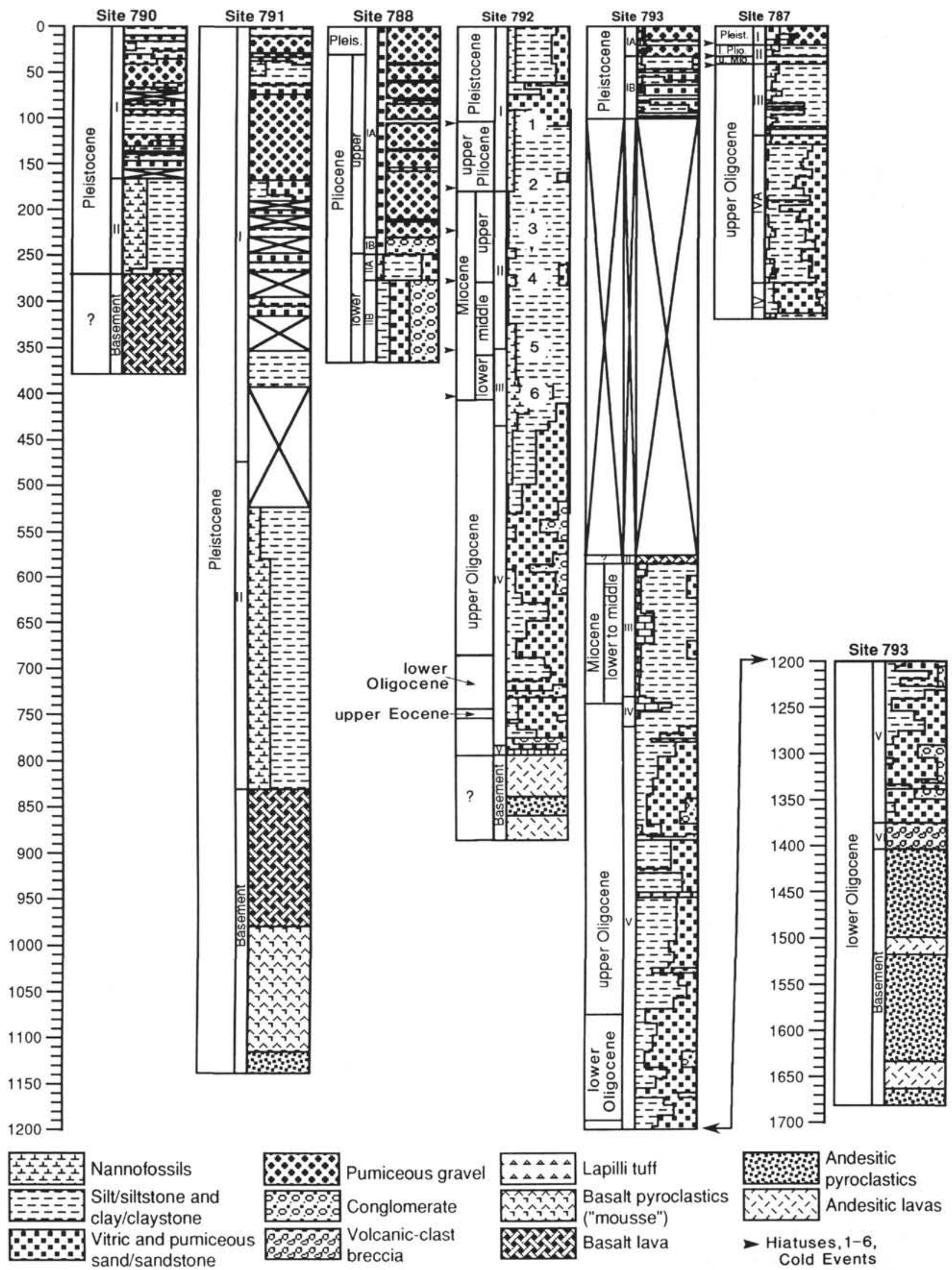

Figure 2. Lithostratigraphy illustrating hiatuses and cold events. 
and 24.5 Ma (between Cores 126-792E-28R and -29R, 396-415 mbsf), Cold Event 6, corresponds to a time of significant enrichment in $8^{18} \mathrm{O}$ in benthic foraminifers (e.g., Miller and Thomas, 1985) and was possibly caused by the opening of the Drake Passage and subsequent development of the deep Circum-Antarctic Current (Keller and Barron, 1983).

The earliest faunas at this site recovered in Cores 126-792B-63R and $-64 \mathrm{R}$ were poorly preserved planktonic foraminifers deposited 37.4-38 Ma.

\section{SUMMARY}

Ocean Drilling Program Leg 126 drilled seven sites in the Izu-Bonin region (Fig. 1) that yielded late Eocene to Quaternary planktonic foraminifers (Fig. 2). Turbidites and volcanic ash beds are intercalated with hemipelagic sediments. Preservation of foraminifers in Quaternary sediments is generally good; however, species abundance varies by a factor of $10^{5}-10^{6}$ and reflects dilution by volcanogenic as well as by terrigenous constituents and cannot be used for paleoceanographic reconstructions. In pre-Quaternary deposits, foraminiferal tests frequently exhibit dissolution effects; biostratigraphic zonation and placement of zonal boundaries is difficult owing to hiatuses, dissolution facies, extraneously deposited sediments, and discontinuous coring. Six hiatuses and/or dissolution periods were recorded at Site 792 (Fig. 2), which may reflect global climatic cooling events and/or changes in oceanic circulation patterns.

\section{ACKNOWLEDGMENTS}

I would like to thank W. A. Berggren for identifying Eocene index species from Site 792 as well as several very poorly preserved Eocene taxa from Site 793 and for confirming the ages suggested by me, George Scott, and N. de B. Hornibrook for very valuable comments of the foraminiferal species. Samples were provided by ODP. This research was partially supported by a grant from the National Science Foundation through subcontract number 20357 to the Texas A\&M Research Foundation.

\section{REFERENCES}

Banner, F. T., and Blow, W. H., 1965. Progress in the planktonic foraminiferal biostratigraphy of the Neogene. Nature, 208:1164-1166.

Barron, J. A., Keller, G., and Dunn, D. A., 1985. A multiple microfossil biochronology for the Miocene. In Kennett, J. P. (Ed.) The Miocene Ocean: Paleoceanography and Biogeography. Geol. Soc. Am. Mem., 163:21-36.

Bé, A.W.H., 1977. An ecological, zoogeographic and taxonomic review of Recent planktonic foraminifera. In Ramsey, A.T.S. (Ed.), Oceanic Micropalaeontology (Vol. 1): New York (Academic Press), 1-100.

Berger, W. H., 1968. Planktonic foraminifera: selective solution and paleoclimatic interpretation. Deep-Sea Res., Part A, 15:31-43

1970. Planktonic foraminifera: selective solution and the lysocline. Mar. Geol., 8:111-138.

, 1973. Deep-sea carbonates: Pleistocene dissolution cycles. J. Foraminiferal Res., 3:187-195.

, 1976. Biogenous deep-sea sediments: production, preservation, and interpretation. In Riley, J. P., and Chester, R. (Eds.), Chemical Oceanography (Vol. 5): London (Academic Press), 265-387.

Berggren, W. A., Kent, D. V., Flynn, J. J., and Van Couvering, J. A., 1985. Cenozoic geochronology. Geol. Soc. Am. Bull., 96:1407-1418.

Bielak, L. E., and Briskin, M., 1978. Pleistocene biostratigraphy, chronostratigraphy and paleocirculation of the southeast Pacific central water core RC 11-220. Mar. Micropaleontol., 3:51-94.

Blanc, P. L., 1981. Paleoclimatologie isotopique et histoire de l'eau profonde Atlantique Depuis 15 millions d'annees [Ph.D. thesis]. Univ. de Paris-Sud (Centre D'Orsay).

Blow, W. H., 1969. Late middle Eocene to Recent planktonic foraminiferal biostratigraphy. In Brönniman, P., and Renz, H. H. (Eds.), Proceedings of the First International Conference on Planktonic Microfossils, Geneva, 1967: Leiden (E. J. Brill), 1:199-422.
Bolli, H. M., and Saunders, J. B., 1985. Oligocene to Holocene low latitude planktonic foraminifera. In Bolli, H. M., Saunders, J. B., and Perch-Nielsen, K. (Eds.), Plankton Stratigraphy: Cambridge (Cambridge Univ. Press), 155-262.

COSOD II, 1987. Rep. Second Conf. Sci. Ocean Drilling. Strasbourg, July 1987.

Coulbourn, W. T., Parker, G. L., and Berger, W. H., 1980. Faunal and solution patterns of planktonic foraminifera in surface sediments of the north Pacific. Mar. Micropaleontol., 5:329-399.

Ericson, D. B., and Wollin, G., 1968. Pleistocene climates and chronology in deep-sea sediments. Science, 162:1227-1234.

Herman, Y., Osmond, J. K., and Somayajulu, B.L.K., 1989. Late Neogene Arctic paleoceanography: micropaleontology, stable isotopes and chronology. In Herman, Y. (Ed.), The Arctic Seas: Climatology, Oceanography, Geology and Biology: New York (Van Nostrand Reinhold), 581-655.

Jenkins, D. G., 1985. Southern mid-latitude Paleocene to Holocene planktic foraminifera. In Bolli, H. M., Saunders, J. B., and Perch-Nielsen, K. (Eds.), Plankton Stratigraphy: Cambridge (Cambridge Univ. Press), 263-282.

Keller, G., 1978. Late Neogene biostratigraphy and paleoceanography of DSDP Site 310 central north Pacific and correlation with the southwest Pacific. Mar. Micropaleontol., 3:97-119.

, 1979a. Early Pliocene to Pleistocene planktonic foraminiferal datum levels in the north Pacific: DSDP Sites 173, 310, and 296. Mar. Micropaleontol., 4:281-294.

, 1979b. Late Neogene paleoceanography of the north Pacific DSDP Sites 173, 310, and 296. Mar. Micropaleontol., 4:281-294.

-, 1980a. Early to middle Miocene planktonic foraminiferal datum levels of the equatorial and subtropical Pacific. Micropaleontology, 26:372-391.

, 1980b. Middle to late Miocene planktonic foraminiferal datum levels and paleoceanography of the north and southeastern Pacific Ocean. Mar. Micropaleontol., 5:249-281.

, 1980c. Planktonic foraminiferal biostratigraphy and paleoceanography of the Japan trench, Leg 57, DSDP. In Scientific Party, Init. Repts. DSDP, 56, 57, Pt. 2: Washington (U.S. Govt. Printing Office), 809-833.

1981a. Miocene biochronology and paleoceanography of the north Pacific. Mar. Micropaleontol., 6:535-551.

, 1981b. The genus Globorotalia in the early Miocene of the equatorial and northwestern Pacific. J. Foraminiferal Res., 11:118-132.

, 1983. Biochronology and paleoclimatic implications of middle Eocene to Oligocene planktic foraminiferal faunas. Mar. Micropaleontol., 7:463-486.

Keller, G., and Barron, J. A., 1983. Paleoceanographic implications of Miocene deep-sea hiatuses. Geol. Soc. Am. Bull., 94:590-613.

Keller, G., Barron, J. A., and Burckle, L. H., 1982. North Pacific late Miocene correlations using microfossils, stable isotopes, percent $\mathrm{CaCO}_{3}$, and magnetostratigraphy. Mar. Micropaleontol., 7:327-357.

Kennett, J. P., and Srinivasan, M. S., 1983. Neogene Planktonic Foraminifera: A Phylogenetic Atlas: Stroudsburg, PA (Hutchinson Ross).

Malmgren, B. A., 1983. Ranking of dissolution susceptibility of planktonic foraminifera from a temperate region of the south Atlantic Ocean. Mar. Micropaleontol., 8:183-191.

Miller, K. G., and Thomas, E., 1985. Late Eocene to Oligocene benthic foraminiferal isotopic record, Site 574, equatorial Pacific. In Mayer, L., Theyer, F., Thomas, E., et al., Init. Repts. DSDP, 85: Washington (U.S. Govt. Printing Office), 771-777.

Nishimura, A., Marsaglia, K. M., Rodolfo, K. S., Collela, A., Hiscott, R. N., Tazaki, K., Gill, J. B., Janecek, T., Firth, J., Isminger-Kelso, M., Herman, Y., Taylor, R. N., Taylor, B., Fujioka, K., and Leg 126 Scientific Party, 1991. Pliocene-Quaternary submarine pumice deposits in the Sumisu Rift area, Izu-Bonin arc. In Fisher, R. V., and Smith, G. A. (Eds.), Sedimentation in Volcanic Settings. Spec. Publ., Soc. Econ. Paleontol. Mineral., 45:201-208.

Parker, F. L., 1967. Late Tertiary biostratigraphy (planktonic foraminifer) of tropical Indo-Pacific deep-sea cores. Bull. Am. Paleontol., 52:115-208.

Pisias, N. G., Shackleton, N. J., and Hall, M. A., 1985. Stable isotope and calcium carbonate records from hydraulic piston cored Hole 574A: highresolution records from the middle Miocene. In Mayer, L., Theyer, F., Thomas, E., et al., Init. Repts. DSDP, 85: Washington (U.S. Govt. Printing Office), $735-748$.

Shackleton, N. J., and Kennett, J. P., 1975. Late Cenozoic oxygen and carbon isotopic changes at DSDP Site 284: implications for glacial history of the Northern Hemisphere and Antarctica. In Kennett, J. P., Houtz, R. E., et al., Init. Repts. DSDP, 29: Washington (U.S. Govt. Printing Office), 801-807. 
Taylor, B., Fujioka, K., et al., Proc. ODP, Init. Repts., 126: College Station, TX (Ocean Drilling Program).

Thompson, P. R., Bé, A.W.H., Duplessy, J. C., and Shackleton, N. J., 1979. Disappearance of pink-pigmented Globigerinoides ruber at 120,000 yr BP in the Indian and Pacific oceans. Nature, 280:554-558.

Thompson, P. R., and Sciarrillo, J. R., 1978. Planktonic foraminiferal biostratigraphy in the equatorial Pacific. Nature, 276:29-33.

Toumarkine, M., and Luterbacher, H., 1985. Paleocene to Eocene planktic foraminifera. In Bolli, H. M., Saunders, J. B., and Perch-Nielsen, K. (Eds.), Plankton Stratigraphy: Cambridge (Cambridge Univ. Press), 87-154.
Williams, D. F., Thunell, R. C., Hodell, D. A., and Vergnaud-Grazzini, C., 1985. Synthesis of Late Cretaceous, Tertiary, and Quaternary stable isotope records of the south Atlantic based on Leg 72 DSDP core material. In Hsü, K. J., and Wiessert, H. J. (Eds.), South Atlantic Paleoceanography: Cambridge (Cambridge Univ. Press), 205-241.

Date of initial receipt: 25 January 1991

Date of acceptance: 20 September 1991

Ms 126B-133 